Journal of Fluid Mechanics

http://journals.cambridge.org/FLM

Journal of

Additional services for Journal of Fluid Mechanics:

Email alerts: $\underline{\text { Click here }}$

Subscriptions: $\underline{\text { Click here }}$

Commercial reprints: $\underline{\text { Click here }}$

Terms of use : $\underline{\text { Click here }}$

\title{
The motion of a singular vortex near an escarpment
}

D. C. DUNN, N. R. McDONALD and E. R. JOHNSON

Journal of Fluid Mechanics / Volume 448 / December 2001, pp 335 - 365

DOI: 10.1017/S0022112001006115, Published online: 26 November 2001

Link to this article: http://journals.cambridge.org/abstract_S0022112001006115

How to cite this article:

D. C. DUNN, N. R. McDONALD and E. R. JOHNSON (2001). The motion of a singular vortex near an escarpment. Journal of Fluid Mechanics, 448, pp 335-365 doi:10.1017/S0022112001006115

Request Permissions : $\underline{\text { Click here }}$ 


\title{
The motion of a singular vortex near an escarpment
}

\author{
By D. C. DUNN†, N. R. McDONALD \\ AND E. R. JOHNSON \\ Department of Mathematics, University College London, Gower Street, \\ London WC1E 6BT, UK
}

(Received 22 June 2000 and in revised form 19 June 2001)

\begin{abstract}
McDonald (1998) has studied the motion of an intense, quasi-geostrophic, equivalentbarotropic, singular vortex near an infinitely long escarpment. The present work considers the remaining cases of the motion of weak and moderate intensity singular vortices near an escarpment. First, the limit that the vortex is weak is studied using linear theory. For times which are short compared to the advective time scale associated with the vortex it is found that topographic waves propagate rapidly away from the vortex and have no leading-order influence on the vortex drift velocity. The vortex propagates parallel to the escarpment in the sense of its image in the escarpment. The mechanism for this motion is identified and is named the pseudoimage of the vortex. Large-time asymptotic results predict that vortices which move in the same direction as the topographic waves radiate non-decaying waves and drift slowly towards the escarpment in response to wave radiation. Vortices which move in the opposite direction to the topographic waves reach a steadily propagating state. Contour dynamics results reinforce the linear theory in the limit that the vortex is weak, and show that the linear theory is less robust for vortices which move counter to the topographic waves. Second, contour dynamics results for a moderate intensity vortex are given. It is shown that dipole formation is a generic feature of the motion of moderate intensity vortices and induces enhanced motion in the direction perpendicular to the escarpment.
\end{abstract}

\section{Introduction}

In the abyssal ocean there are regions where, at least locally, the background gradient of potential vorticity is dominated by varying topography, rather than the ubiquitous planetary curvature, or the $\beta$-effect as it is often represented in theoretical models. Of interest to the present study are regions of sharp topographic gradients such as the mid-Atlantic ridge, or the continental margins. In such regions the trajectories of eddies, such as those affecting the dispersal of newly formed bottom water, are expected to depend, to leading order, on the local topographic gradients. See for example Stern (2000), Beismann, Käse \& Lutejeharms (1999) and Richardson \& Tychensky (1998). Atmospheric eddies, such as tropical cyclones are also affected by sharp topographic gradients such as coastal mountain barriers. Given the potential damaged caused by cyclones impinging on inhabited areas, the calculation of their trajectories is an important meteorological question.

$\dagger$ Present address: Department of Aeronautics, Imperial College, Prince Consort Road, London SW7 2AZ, UK. 
In a previous study McDonald (1998) investigated the motion of an intense singular vortex near an escarpment, using quasi-geostrophic $f$-plane dynamics. The vortex is defined as intense if the time scale for the vortex circulation is much shorter than the time scale for topographic wave generation, so that advection of fluid across the escarpment dominates over topographic wave generation. Under this assumption it was found that, initially, cyclones propagate away from the deep-water region and anticyclones propagate away from the shallow-water region. Asymptotic results showed that eventually cyclones and anticyclones propagate parallel to the escarpment at a speed that decays exponentially with the distance of the vortex from the escarpment. Moreover the drift speed always matches a possible topographic phase speed, and non-decaying topographic waves are radiated as a result. The vortex responds to this radiation by drifting slowly perpendicular to the escarpment. Provided that they are initially located within about a Rossby radius from the escarpment, cyclones accumulate at a distance of about 1.2 Rossby radii from the escarpment on the shallow side. Anticyclones exhibit similar behaviour except that they accumulate on the deep side of the escarpment.

As well as being important in its own right, the escarpment topography serves as a useful paradigm for the $\beta$-plane. In particular the direction going from deep to shallow water is analogous to the northwards direction on the $\beta$-plane, since both these directions are in the sense of increasing ambient potential vorticity. The behaviour of an intense singular vortex near an escarpment is qualitatively the same as the behaviour of an intense singular vortex on the $\beta$-plane, studied by Reznik (1992). Moreover the same curved trajectories for non-singular, intense $\beta$-plane vortices have been observed in a number of laboratory (e.g. Firing \& Beardsley 1976), numerical (e.g. McWilliams \& Flierl 1979; Mied \& Lindeman 1979; Lam \& Dritschel 2001) and analytic studies (e.g. Flierl 1984; Sutyrin \& Flierl 1994; Reznik, Grimshaw \& Benilov 2000).

The aim of the present study is to examine the motion of weak and moderate singular vortices near an escarpment. It should be noted that there is no analogy of a weak singular vortex on a $\beta$-plane, since the Bessel function structure of a singular vortex implies that near the vortex centre the swirl velocity becomes arbitrarily large and advective effects must locally dominate the dispersive effects of $\beta$. Recently Lam \& Dritschel (2001) have applied the high-resolution Contour Advective semiLagrangian (CASL) algorithm of Dritschel \& Ambaum (1997) to the study of an initially circular vortex on the $\beta$-plane. It was found the moderate vortices undergo enhanced meridional drift due to dipole formation. Similar behaviour has been observed for barotropic vortices near an escarpment in recent laboratory experiments by Zavala Sanson, van Heijst \& Doorschoot (2000). It was shown that moderate intensity anticyclones located on the shallow side of the escarpment are able to "climb" the topographic gradient, while cyclones located on the shallow side are "back-reflected".

The paper is organized as follows. First the motion of a weak singular vortex is investigated using linear theory. Analytical solutions are found using Fourier analysis. The results of linear theory are then tested through a contour dynamics investigation. Second, the motion of moderate intensity singular vortices, for which no analytic theory is available, is investigated using contour dynamics. It is found that dipole formation is characteristic for moderate vortex interactions. Finally the conclusions of the study are presented. 


\section{Problem formulation}

Shallow-water, quasi-geostrophic motion on the $f$-plane is governed by the conservation of potential vorticity, which can be written in non-dimensional form as

$$
\frac{\partial}{\partial t}\left(\nabla^{2} \psi-\psi\right)+J\left[\psi, \nabla^{2} \psi-\psi\right]+S \frac{\partial \psi}{\partial x} \frac{\partial h_{B}}{\partial y}=0,
$$

where the conserved quantity is the quasi-geostrophic potential vorticity,

$$
Q=\nabla^{2} \psi-\psi+\operatorname{Sh}_{B}(y) .
$$

Here $\psi$ is the streamfunction, $t$ is the time, $h_{B}(y)$ is the topography which is assumed to only vary in the $y$-direction and $J[f, g]=f_{x} g_{y}+g_{x} f_{y}$ is the Jacobian. Note that the form of (2.2) implies that equivalent-barotropic dynamics are assumed. In the context of the present work this is an attempt to model the stratification of the abyssal ocean, i.e. a layer of dense fluid lying under a layer of less dense fluid, and where the interface between the two layers is free to deform. The length scale used in this non-dimensionalization is the Rossby radius $R_{D}=\left(g^{\prime} D\right)^{1 / 2} / f$, where $D$ is the average depth of the layer containing the vortex, $g^{\prime}$ is the acceleration due to the reduced gravity of the fluid and $f$ is the Coriolis parameter. The non-dimensional time scale is the eddy turnover or advective time scale, $T_{a}=R_{D} / U=D f / \Lambda$, where $\Lambda$ is the scale for the vortex amplitude and $U$ is the typical vortex velocity due to geostrophy. The parameter $S$ is given by

$$
S=\frac{R_{D} / U}{\delta^{-1} f^{-1}}=\frac{\delta}{R o}=\frac{T_{a}}{T_{w}},
$$

where $\delta$ is the scale height of the topography, so that $T_{w}$ is the time scale for topographic wave generation (see, for example, Johnson 1984). The two numbers $\delta$ and $R o$ are small parameters, but their ratio $S$ may take the whole range of values. The Rossby number, $R o$, is a function of the distance of the vortex from the escarpment, i.e. the critical velocity $U$ is the velocity induced by the vortex at the escarpment rather than the absolute value of the circulation. This can be estimated from the structure of the vortex. For example, in this work, this can be done using the Bessel function structure of the singular vortex. McDonald (1998) has studied the case $S \ll 1$, where the effect of the topography is relatively weak and the vortex is said to be intense. The present work considers the remaining cases, $S \gg 1$ where the effect of the topography is strong, i.e. a weak vortex, and $S \approx 1$, where the effect of the topography and the vortex are comparable, i.e. a moderate vortex.

In the absence of topography, i.e. $h_{B}(y)=0$, isolated singular vortex solutions to (2.1) are well known and are

$$
\Psi_{v}(x-X, y-Y)=-\frac{\Gamma}{2 \pi} K_{0}\left(\left((x-X)^{2}+(y-Y)^{2}\right)^{1 / 2}\right),
$$

where $(X(t), Y(t))$ is the position of the vortex centre, and $K_{0}$ the modified Bessel function of the first kind, order zero. Such solutions are obtained by solving

$$
\nabla^{2} \Psi-\Psi=\Gamma \delta(x-X, y-Y) .
$$

The sign of $\Gamma$ gives the sense of the circulation: for $\Gamma<0$ it is clockwise (anticyclonic) and for $\Gamma>0$ it is anticlockwise (cyclonic). By integrating (2.1) over all space, and provided $\psi \rightarrow 0$ sufficiently rapidly in the far field, it straightforward to show that $\mathrm{d} \Gamma / \mathrm{d} t=0$, i.e. $\Gamma=$ constant.

The present work considers the initial value problem for a singular vortex in the 

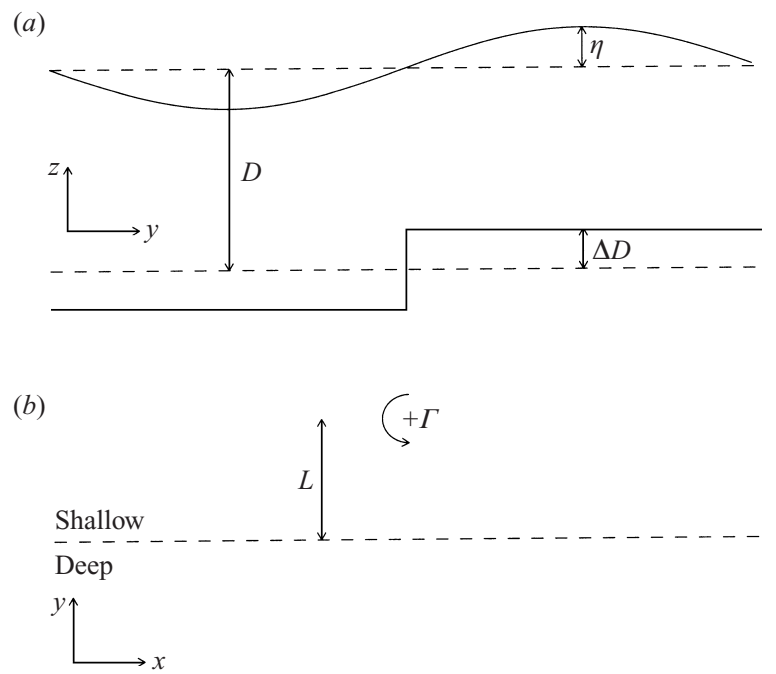

FIGURE 1. The present problem. (a) A plot of the cross-section of the fluid domain, and the dimensional variables. The free surface elevation, $\eta$ is scaled on $\operatorname{Rof} L^{2}$ and written $\psi ; D$ is the typical layer depth in the absence of motion, and $\delta=\Delta D / D$ is half the fractional height of the topography. (b) The initial condition, which is a vortex (here a cyclone, indicated by + , and with strength $\Gamma>0$ ), distance $L$ from the escarpment, which is aligned along $y=0$. Shallow water lies in the half-plane $y>0$, and so contains fluid with high ambient potential vorticity.

presence of non-zero topography. Figure $1(a)$ shows the present choice of topography, an infinitely long escarpment aligned along $y=0$, which can be expressed as

$$
h_{B}(y)=\operatorname{sgn}(y) .
$$

By analogy with the $\beta$-plane, shallow fluid lies in the direction of increasing $y$. For convenience of description, the direction of increasing $y$ is identified as north, and increasing $x$ as east. In reality there is no preferential direction on the $f$-plane, so this choice is made simply so as to align the isobaths, or potential-vorticity contours, in the $\beta$-plane sense. The fluid motion is strictly not quasi-geostrophic near $y=0$, since there the topography has infinite gradient, so the flow must be three-dimensional near the escarpment. However, the present choice of topography is an attempt to model a sudden depth change, $\Delta D$, i.e. the horizontal length scale of the depth change (say $l$ ) is such that $R_{D} \gg l \gg \Delta D$. This implies that the vertical velocity scales like $\Delta D / l \ll 1$, and it is assumed that the three-dimensional effects near the escarpment are negligible and have no leading-order effect on the dynamics.

Johnson \& Davey (1990) have shown that the dispersion relation for the linear topographic waves $(S \gg 1)$ in this domain is

$$
\omega=-\frac{S k}{\sqrt{k^{2}+1}}
$$

and the phase and group velocities are given by

$$
\begin{aligned}
& c_{p}(k)=-\frac{S}{\sqrt{k^{2}+1}}, \\
& c_{g}(k)=-\frac{S}{\left(k^{2}+1\right)^{3 / 2}},
\end{aligned}
$$


so both the phase and energy of the waves propagate in the direction of decreasing $x$, i.e. with shallow water to the right.

At time $t=0$ the vortex has location $(X(0), Y(0))=(0, L)$, as shown in figure $1(b)$. For $t>0$ the vortex advects fluid columns across the isobaths, demanding an associated change in their relative vorticity. This establishes secondary circulations which advect the primary vortex. The aim is to determine the vortex trajectory for $t>0$, i.e. $X(t)$ and $Y(t)$. In the following sections the solution for a weak vortex is found by Fourier analysis; the form of the solution also enables a simple approximation for the mass transport by the vortex to be made. Contour dynamics simulations are used to investigate the motion of a moderate vortex. Finally the results and limitations of the present model are discussed.

\section{A weak singular vortex}

For a weak vortex $S \gg 1$. Set $\epsilon=S^{-1} \ll 1$. Seeking a solution of the form

$$
\psi=\Psi_{v}(x-X, y-Y)+\phi,
$$

the governing equation (2.1) becomes

$$
\epsilon\left(\nabla^{2} \phi-\phi\right)_{t}+\epsilon J\left[\phi, \nabla^{2} \phi-\phi\right]+\epsilon J\left[\Psi_{v}, \nabla^{2} \phi-\phi\right] \frac{\partial \phi}{\partial x} \frac{\partial h_{B}}{\partial y}=-\frac{\partial \Psi_{v}}{\partial x} \frac{\partial h_{B}}{\partial y} .
$$

\subsection{Short-time solution}

Introduce the rescaled time variable $\tau=t / \epsilon$, so that the unit of time is the short, topographic-wave time scale $T_{w}$, and denote the solution on this time scale by $\phi_{0}$. For $\tau \ll O\left(\epsilon^{-1}\right)$ the advection term in (3.2) is negligible, so for times up to $\tau=O\left(\epsilon^{-1}\right)$,

$$
\left(\nabla^{2} \phi_{0}-\phi_{0}\right)_{\tau}+\frac{\partial \phi_{0}}{\partial x} \frac{\partial h_{B}}{\partial y}=-\frac{\partial \Psi_{v}}{\partial x} \frac{\partial h_{B}}{\partial y},
$$

which is a linear, forced topographic wave equation, the forcing being due to the vortex. On this time scale the vortex drift velocity components are $O(\epsilon)$, and advection of the vortex is by the regular $\phi_{0}$-field so that

$$
\begin{aligned}
& \frac{\mathrm{d} X}{\mathrm{~d} \tau}=-\left.\epsilon \frac{\partial \phi_{0}}{\partial y}\right|_{x=X, y=Y}, \\
& \frac{\mathrm{d} Y}{\mathrm{~d} \tau}=\left.\epsilon \frac{\partial \phi_{0}}{\partial x}\right|_{x=X, y=Y} .
\end{aligned}
$$

Hence, for times up to $\tau=O\left(\epsilon^{-1}\right), X=O(\epsilon)$ and $Y=L+O(\epsilon)$, and the vortex term is, to leading order

$$
\Psi_{v}=\Psi_{v}(x, y-L),
$$

on this time scale. Also $\partial h_{B} / \partial y=2 \delta(y)$ so away from $y=0(3.3)$ is

$$
\nabla^{2} \phi_{0}-\phi_{0}=0 \text {. }
$$

The topography is 'switched on' near a pre-existing vortex at $\tau=0$, i.e the initial condition is

$$
\phi_{0}(x, y, 0)=0 .
$$

The boundary conditions are

$$
\nabla \phi_{0} \rightarrow 0, \quad x^{2}+y^{2} \rightarrow \infty
$$




$$
\begin{gathered}
{\left[\phi_{0}\right]=0, \quad y=0,} \\
{\left[\phi_{0 y t}\right]+2 \phi_{0 x}=-2 \Psi_{v x}(x,-L), \quad y=0,}
\end{gathered}
$$

where [.] denotes the jump of the enclosed quantity across the escarpment. Condition (3.9) is the requirement that the fluid be at rest far from the escarpment, while (3.10) and (3.11) are obtained by integrating the governing equations across the escarpment. Similar linear initial-boundary value problems for the escarpment topology have previously been considered by, among others, Johnson (1985), Johnson \& Davey (1990) and McDonald (1992, 1996), where details of the derivation of the boundary conditions and the solution procedure can be found. The solution to this problem is obtained through standard Fourier transform methods. It is straightforward to show that the solution consists of a steady term and a topographic wave term,

$$
\phi_{0}=\phi_{0}^{(s)}+\phi_{0}^{(w)}
$$

where

and

$$
\phi_{0}^{(s)}=\frac{\Gamma}{2 \pi} K_{0}\left(\left(x^{2}+(|y|+|L|)^{2}\right)^{1 / 2}\right)
$$

$$
\phi_{0}^{(w)}=-\frac{\Gamma}{2 \pi} \int_{0}^{\infty} A(k, y) \cos (k x-\omega \tau) \mathrm{d} k
$$

with

$$
A(k, y)=\frac{\exp \left(-(|y|+|L|) \sqrt{k^{2}+1}\right)}{\sqrt{k^{2}+1}} .
$$

In obtaining (3.13) an identity from Gradshteyn \& Ryzhik (1980, p. 498), has been used. At $\tau=0$ the topographic wave term $\phi_{0}^{(w)}$ cancels with the steady term $\phi_{0}^{(s)}$, giving the correct initial condition.

\subsubsection{The steady term}

The steady term $\phi_{0}^{(s)}$ is reminiscent of an image of the vortex in the escarpment $y=0$. Note however the important distinction: if $L<0$ then $\Psi_{v}+\phi_{0}^{(s)}$ vanishes for $y>0$ and similarly if $L>0$ then $\Psi_{v}+\phi_{0}^{(s)}$ is zero for $y<0$. Thus the fluid on the same side of the escarpment as the vortex feels the effect of an image vortex in the escarpment, whereas the fluid across the escarpment from the vortex is undisturbed with respect to the steady term, $\Psi_{v}+\phi_{0}^{(s)}$. For this reason the steady term, $\phi_{0}^{(s)}$, is dubbed the pseudoimage of the vortex. The streamlines of $\Psi_{v}+\phi_{0}^{(s)}$ are shown in figure 2 .

The role of the pseudoimage in the following theory is vital. In the contour dynamics investigations described below, it will be seen that the behaviour of the vortex is predicted well by the pseudoimage description for many eddy turnover times. It should be emphasized that the pseudoimage has a definite physical meaning. It is part of the topographic wavetrain, a wavetrain which is initially excited by the circulation of the vortex pushing fluid across the escarpment. It is non-dispersive and remains localized near the vortex, is even in $y$, and is not singular anywhere. The magnitude of the relative vorticity associated with the disturbance $\phi_{0}^{(s)}$ is precisely that required to advect the vortex as if the escarpment were a plane wall. Importantly, it will be shown that the dispersive topographic waves rapidly propagate away from the vortex, and have no influence on it for times $\tau \rightarrow \epsilon^{-1}$, i.e. for large times the advection of the vortex is due solely to its pseudoimage. The properties of the dispersive waves are discussed next. 


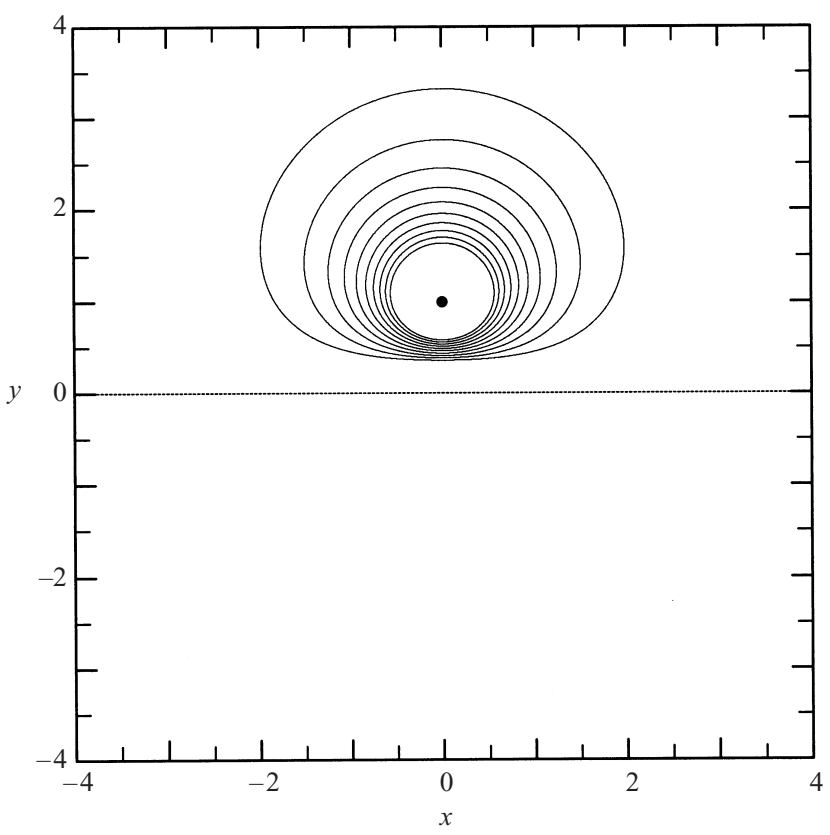

FIgURE 2. Streamlines for the steady term $\psi_{\text {stat }}$. The vortex has unit strength and is located at $(0,1)$. The contour interval is 0.01 , and the position of the escarpment is indicated by the solid line. Note the closed streamlines on the side of the escarpment occupied by the vortex. The fluid on the opposite side is undisturbed.

\subsubsection{The topographic wave term}

The unsteady part of the solution $\phi_{0}^{(w)}$ given by (3.14) is a Fourier superposition of topographic waves. The amplitude is maximum over $y=0$, coinciding with the escarpment. The dispersion relation is given by (2.7) and the phase and group velocities are given by equations (2.8) and (2.9) respectively.

Standard asymptotic methods are employed to deduce the large-time behaviour of $\phi_{0}^{(w)}$, as in Johnson (1985). The integrand in (3.14) is analytic, so the only contributions to the integral as $\tau \rightarrow \infty$ come from the points of stationary phase (see, for example, Bender \& Orszag 1978). In the present case there is only one point of stationary phase, which occurs for $-\tau<x<0$, and is given by

$$
k_{s}=\left((-\xi)^{-2 / 3}-1\right)^{1 / 2},
$$

where $\xi=x / \tau$. It is straightforward to show that

$$
\phi_{0}^{(w)} \approx \frac{\Gamma}{\sqrt{2 \pi}} \exp \left(-(|y|+|L|) \sqrt{k_{s}^{2}+1}\right)\left(\frac{1}{3 \tau \xi k_{s}}\right)^{1 / 2} \cos \left(k_{s} x-\omega_{s} \tau-\frac{\pi}{4}\right),
$$

where $\omega_{s}=-k_{s}\left(k_{s}+1\right)^{-1 / 2}$. The solution $\phi_{0}^{(w)}$ decays like $\tau^{-1}$ for any fixed $x$ and it decays like $\tau^{-1 / 2}$ for any fixed $\xi=x / \tau,-1<\xi<0$.

The stationary phase approximation breaks down at the point $x=-\tau$, corresponding to the point of maximum group velocity $\omega^{\prime \prime}(k)=c_{g}^{\prime}(k)=0$. Following Lighthill (1974), a third-order expansion of the phase gives, near $x=-\tau$, as $\tau \rightarrow \infty$

$$
\phi_{0}^{(w)} \approx \frac{\Gamma}{2} \frac{\mathrm{e}^{-(|y|+|L|)}}{(3 \tau / 2)^{1 / 3}} \cos \tau \mathrm{Ai}\left(-\frac{x-\tau}{(3 \tau / 2)^{1 / 3}}\right)
$$



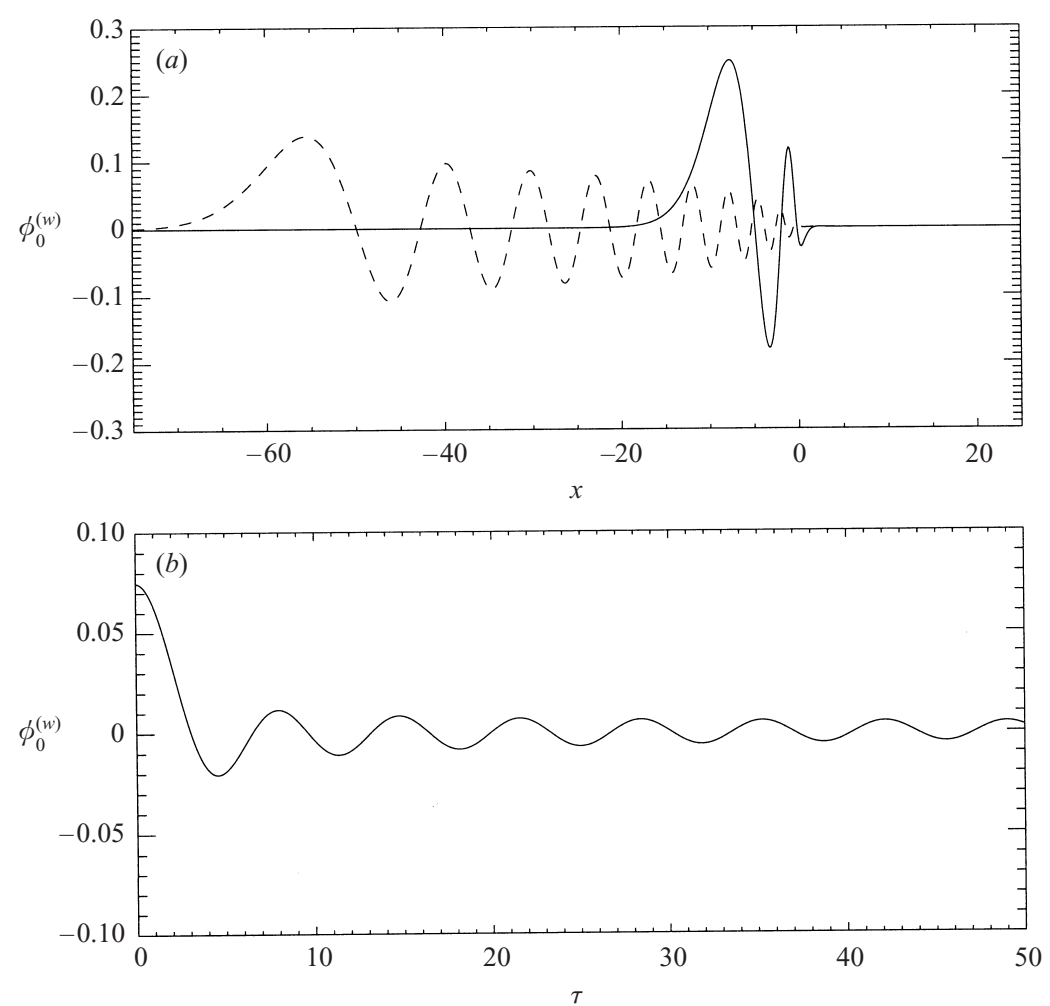

Figure 3. (a) Plot of $\phi_{0}^{(w)}$ evaluated over $y=0$. Times are $\tau=10$ (solid curve) and $\tau=60$ (dashed curve). (b) The wave term evaluated at the vortex centre as a function of $\tau$. In each case $\Gamma=1$, i.e. an anticyclone, and the solution has been scaled by $2 \pi$. See text for further comments.

where Ai is the Airy function (see Abramowitz \& Stegun 1972 for details). Thus, $\phi_{0}^{(w)}$ decays exponentially for $x<-\tau$ and oscillates for $x>-\tau$. The amplitude of this maximum oscillation at the wavefront decays like $\tau^{-1 / 3}$, and the wavefront approximation smoothly matches the topographic waves to the undisturbed fluid ahead of the train (see Lighthill 1974 for details). The wave term evaluated over $y=0$ is illustrated in figure $3(a)$ at two different times, $\tau=10$ and 60 . The plot is obtained by numerical integration of (3.14). The westward propagating topographic waves are evident. The maximum disturbance at the wavefront coincides with the $x$-location of the vortex. The magnitude of this maximum amplitude decreases with time. It is also evident that the amplitude of the disturbance at $x=0$ decreases more rapidly than the maximum amplitude of the wavetrain.

Of particular interest is the influence of the waves at the vortex centre. The wave amplitude decays most rapidly at the $x$-location of the vortex centre, and this can be seen as follows. There are no points of stationary phase for $x=0$. Equation (3.14) evaluated at the vortex centre is

$$
\phi_{0}^{(w)}(0, L, \tau)=-\frac{\Gamma}{2 \pi} \int_{0}^{\infty} \frac{\exp \left(-2|L| \sqrt{k^{2}+1}\right)}{\sqrt{k^{2}+1}} \cos \frac{k \tau}{\sqrt{k^{2}+1}} \mathrm{~d} k .
$$

Writing $\xi=k / \sqrt{k^{2}+1}$ this may be rewritten

$$
\phi_{0}^{(w)}(0, L, \tau)=-\frac{\Gamma}{2 \pi} \int_{0}^{1} h(\xi) \cos \xi \tau \mathrm{d} \xi
$$


where

$$
h(\xi)=\frac{\exp \left(-2|L| / \sqrt{1-\xi^{2}}\right)}{\sqrt{1-\xi^{2}}} .
$$

The hypotheses of the Riemann-Lebesgue Lemma (e.g. Bender \& Orszag 1978) are satisfied and so the integral in (3.20) decays like $\tau^{-1}$ as $\tau \rightarrow \infty$. Hence, the influence of the waves on the vortex decreases algebraically for large $\tau$. Figure 3(b) shows a plot of the response at the vortex centre, and has been obtained by numerical integration of (3.19).

Importantly, as $\tau \rightarrow \epsilon^{-1}$ the waves have no influence on the vortex drift velocity. The trajectory of the vortex centre on the topographic wave time scale is considered in detail in the next subsection.

\subsubsection{Short-time vortex trajectory}

First consider times $\tau \ll 1$. The solution (3.12) is, to leading order in $\tau$,

$$
\phi_{0} \approx-\frac{\Gamma \tau}{2 \pi} \int_{0}^{\infty} \omega A(k, y) \sin k x \mathrm{~d} k
$$

as $\tau \rightarrow 0$. The pseudoimage term cancels with the $\cos k x$ term in the expansion of the wave term, and so the pseudoimage has no leading-order effect on the vortex at initial times. The vortex velocity components given by (3.4) are then

$$
\begin{aligned}
& \frac{\mathrm{d} X}{\mathrm{~d} \tau}=0 \\
& \frac{\mathrm{d} Y}{\mathrm{~d} \tau}=-\frac{\epsilon \Gamma \tau}{2 \pi} \int_{0}^{\infty} \omega k A(k, L) \mathrm{d} k .
\end{aligned}
$$

The integral in (3.24) converges, since $|A(k, y)|<\mathrm{e}^{-k}$, and, moreover, is negative, since $\omega$ is negative for $k>0$. Hence, for $\tau \ll 1$ the vortex moves with velocity increasing linearly in time, in the $y$-direction. Cyclones $(\Gamma>0)$ drift north and anticyclones $(\Gamma<0)$ move south, regardless of the sign of $L$.

For times $1 \ll \tau<\epsilon^{-1}$. The topographic waves have propagated away and have no influence on the vortex. Then, the advection of the vortex is due solely to the pseudoimage. From equations (3.4) and (3.5)

$$
\frac{\mathrm{d} X}{\mathrm{~d} \tau}=\epsilon u, \quad \frac{\mathrm{d} Y}{\mathrm{~d} \tau}=0,
$$

where

$$
u=\frac{\Gamma}{2 \pi} K_{1}(2|L|) \operatorname{sgn} L .
$$

Anticyclones $(\Gamma<0)$ move west (resp. east) in shallow (deep) water. Cyclones $(\Gamma>0)$ move east (resp. west) in shallow (deep) water. Bell (1989) and Stern \& Flierl (1987) find that weak barotropic (i.e. 'log') vortices near a potential-vorticity interface due to piecewise-constant shear flow also exhibit this behaviour.

To understand the physical mechanism responsible for the drift of the vortex, consider figure 4 . This plot shows the evolution of the streamlines associated with the $\phi_{0}$-field, for $\Gamma=-1$, i.e. an anticyclone. In figure 4(a), the streamlines of the short-time solution given by equation (3.22) are plotted. The initial response is the establishment of a secondary dipole, centred over the escarpment and with its axis aligned along the $y$-axis. This is the result of the anticyclone drawing fluid to its 

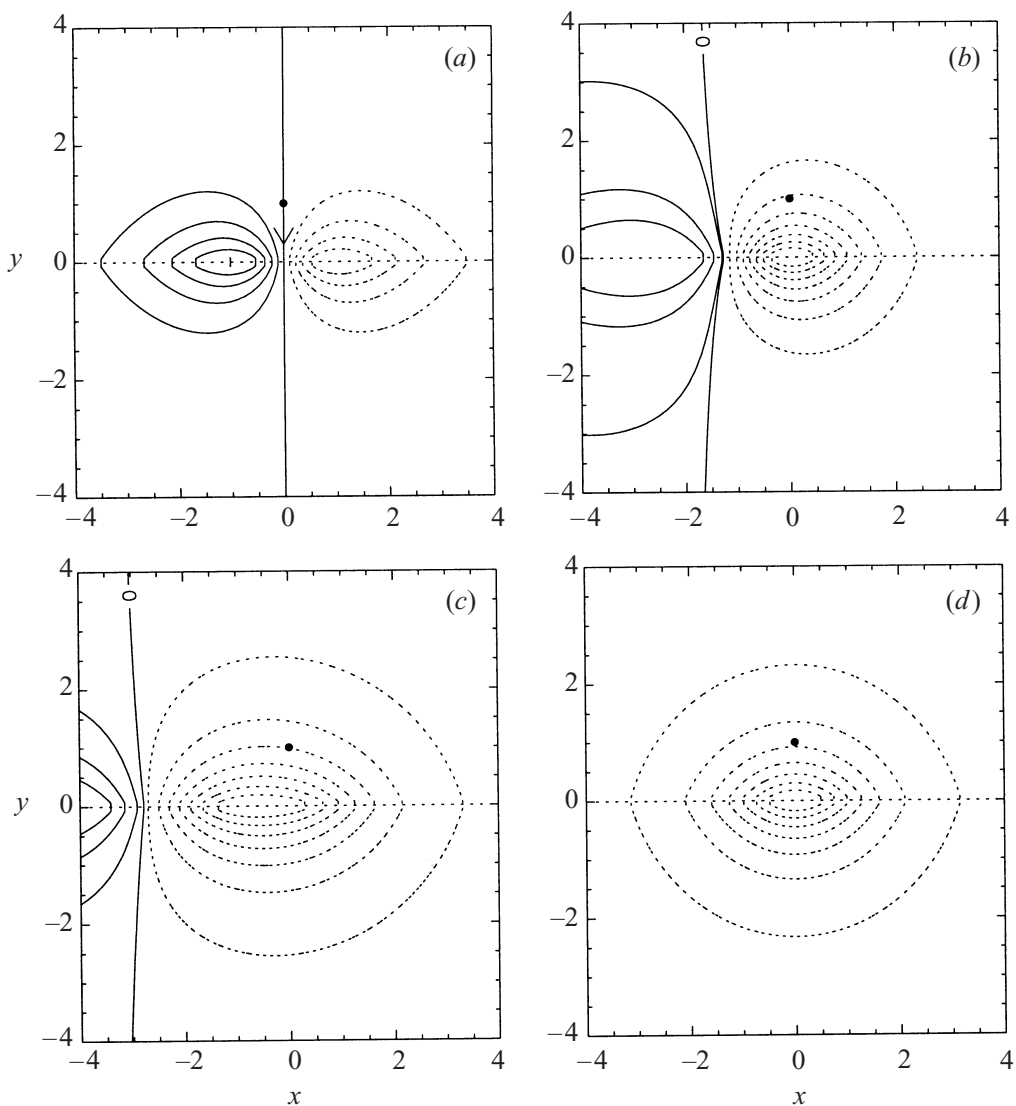

FIGURE 4. The advection of the vortex, here an anticyclone shown by the black dot, by the regular field. (a) Evaluation of $\phi$ at $\tau=0^{+}$, by equation (3.22). The contour values have been scaled by $\tau / 2 \pi$. Note the initial advection is south, along the dipole axis. (b) $\phi$ evaluated at $\tau=4$ and $(c)$ at $\tau=7$, from equation (3.12). (d) The pseudoimage term, the regular field as $\tau \rightarrow \infty$. The dashed contours are negative $\phi_{0}$. See text for further comments.

west from the deep side of the escarpment, and pushing fluid to its east away from the shallow side of the escarpment. The vortex moves south along the dipole axis. $\uparrow$ These secondary circulations, induced by the circulation of the primary vortex, diminish rapidly, as energy is lost to the topographic waves. This process is clear in figure $4(b, c)$, which show the rapid westward propagation of the topographic waves, away from the vortex centre. The final frame, figure $4(d)$, shows that as $\tau \rightarrow \infty$ all that remains is the non-dispersive pseudoimage term. Note also that the simple structure of the solution enables an approximation for the mass transport due to the pseudoimage. See Meleshko et al. (1992) for details concerning the calculation of the size of a dipole atmosphere. Note in particular that the mass transport is related to the distance of the vortex from the escarpment.

This behaviour is in contrast to the motion of an intense singular vortex near an escarpment. McDonald (1998) showed that intense cyclones move northwest, while

$\dagger$ This is precisely the initial response for an intense vortex near an escarpment and on a $\beta$-plane. In those cases however the strong circulation of the vortex rotates the dipole axis before the initial disturbance can disperse as topographic waves, causing the southwest (northwest) curved trajectories for anticyclones (cyclones). 
intense anticyclones move southwest. Importantly, both cyclones and anticyclones approach a steady westward drift velocity that matches a possible topographic-wave phase velocity, so that wave radiation must eventually become an important factor in the motion of the vortex. In the present weak limit this is not always the case as, for instance, shallow-water cyclones and deep-water anticyclones move east, i.e. antiparallel to the topographic waves. The large-time response of the vortex is the subject of the following section.

\subsection{Large-time solution}

The adjustment on the short, topographic-wave time scale is assumed to happen instantaneously on the advective time scale. That is, the large- $\tau$ asymptotic steady solution of the previous subsection is taken as the initial condition for the slower, advective adjustment. Denote by $\phi_{1}$ the solution to (3.2) on the advective time scale. The two solutions are then matched by demanding that

$$
\lim _{t \rightarrow 0^{+}} \phi_{1}=\lim _{\tau \rightarrow \infty} \phi_{0}=\Psi_{v}(x,|y|+|L|),
$$

from equations (3.12), (3.13) and (3.14). For times $t \ll 1$, from equation (3.26) the vortex centre moves with the steady drift velocity $X(t)=u t$, suggesting a solution of the form

$$
\psi(x, y, t)=\Psi_{v}(x-u t, y-L)+\phi_{1}(x, y, t) .
$$

Note that $J\left[\nabla^{2} \phi_{0}, \phi_{0}\right]=0$ in equation (2.1) and hence, the response is linear as $t \rightarrow 0^{+}$. It is assumed that this remains the case for finite $t$. This assumption is justified $a$ posteriori, by contour dynamics investigations of the full nonlinear problem.

The linear governing equation for $\phi_{1}$ is then

$$
\epsilon\left(\nabla^{2} \phi_{1}-\phi_{1}\right)_{t}+\epsilon J\left(\Psi_{v}, \nabla^{2} \phi_{1}-\phi_{1}\right)+\frac{\partial \phi_{1}}{\partial x} \frac{\partial h_{B}}{\partial y}=-\frac{\partial \Psi_{v}}{\partial x} \frac{\partial h_{B}}{\partial y} .
$$

Away from $y=0$ this is

$$
\nabla^{2} \phi_{1}-\phi_{1}=0 .
$$

The initial condition is given by (3.27) and the boundary conditions obtained as above are

$$
\begin{gathered}
\nabla \phi_{1} \rightarrow 0, \quad x^{2}+y^{2} \rightarrow \infty, \\
{\left[\phi_{1}\right]=0, \quad y=0,} \\
\epsilon\left[\phi_{1 y t}\right]+2 \phi_{1 x}=-2 \Psi_{v x}(x-u t,-L), \quad y=0 .
\end{gathered}
$$

It has been assumed that the vortex moves predominantly along the escarpment with the velocity given by (3.26), i.e. in order to be self-consistent the vortex drift velocity generated by the solution $\phi_{1}$ should match that given by (3.26). That this is the case is shown below. Denoting the Fourier transform of $\phi_{1}$ by $\hat{\phi}_{1}$, it is straightforward to show that

$$
\hat{\phi}_{1}(k, y, t)=\frac{\Gamma}{2 \sqrt{k^{2}+1}} \exp \left(-(|y|+|L|) \sqrt{k^{2}+1}\right) \frac{1}{k u-\omega}\left(k u \mathrm{e}^{-\mathrm{i} \omega t}-\omega \mathrm{e}^{-\mathrm{i} k u t}\right) .
$$

where

$$
\omega=-\frac{k}{\epsilon \sqrt{k^{2}+1}}
$$

is the topographic wave frequency on the advective time scale. The solution consists 
of a quasi-steady term and a topographic wave term, and can be written

$$
\phi_{1}(x, y, t)=\phi_{1}^{(s)}+\phi_{1}^{(w)}
$$

where

$$
\begin{aligned}
& \phi_{1}^{(s)}=\frac{\Gamma}{4 \pi} \int_{C} \frac{\exp \left(-(|y|+|L|) \sqrt{k^{2}+1}\right)}{1+\epsilon u \sqrt{k^{2}+1}} \frac{1}{\sqrt{k^{2}+1}} \mathrm{e}^{\mathrm{i} k(x-u t)} \mathrm{d} k, \\
& \phi_{1}^{(w)}=\frac{\Gamma \epsilon u}{4 \pi} \int_{C} \frac{\exp \left((-|y|+|L|) \sqrt{k^{2}+1}\right)}{1+\epsilon u \sqrt{k^{2}+1}} \mathrm{e}^{\mathrm{i}(k x-\omega t)} \mathrm{d} k .
\end{aligned}
$$

The $\phi_{1}^{(s)}$ term is a non-dispersive term of the form $\phi_{1}^{(s)}=\phi_{1}^{(s)}(x-u t, y)$, and so is a disturbance which propagates with the vortex. To leading order in the binomial expansion with respect to $\epsilon$ of the integrand this term is the steadily propagating pseudoimage,

$$
\phi_{1}^{(s)}=\frac{\Gamma}{2 \pi} K_{0}\left(\left((x-u t)^{2}+(|y|+|L|)^{2}\right)^{1 / 2}\right) .
$$

Moreover the topographic wave term is $O(\epsilon)$, so that the leading-order vortex drift velocity along the escarpment generated by (3.36) is

$$
\frac{\mathrm{d} X}{\mathrm{~d} t}=-\left.\frac{\partial \phi_{1}^{(s)}}{\partial y}\right|_{x=u t, y=L}=\frac{\Gamma}{2 \pi} K_{1}(2|L|) \operatorname{sgn} L,
$$

i.e. the long-escarpment drift is consistent with (3.26). The large-time drift perpendicular to the escarpment is calculated below.

The second term $\phi_{1}^{(w)}$ is a Fourier superposition of topographic waves. The dispersion relation is given by (3.35), and so the phase and group velocities are

$$
\begin{aligned}
& c_{p}(k)=-\frac{1}{\epsilon \sqrt{k^{2}+1}}, \\
& c_{g}(k)=-\frac{1}{\epsilon\left(k^{2}+1\right)^{3 / 2}}
\end{aligned}
$$

as before. Note that the appearance of $\epsilon$ in these quantities is due to the time scale being the advective time scale.

Each of (3.37) and (3.38) has simple poles for $k= \pm \gamma$ where

$$
\gamma=\left(\frac{1}{\epsilon^{2} u^{2}}-1\right)^{1 / 2}
$$

whenever $-1<\epsilon u<0$, i.e. whenever the vortex drifts west. In order that the waves radiate away from the vortex the inversion contour, $C$, must pass below these poles. The existence of poles gives rise to the possibility of a steady wavetrain as $t \rightarrow \infty$. There are only two cases to consider since the case $u=0$ is of no interest because there is no vortex, and the cases $|\epsilon u| \geqslant 1$ are ruled out, otherwise $u$ is an $O(1 / \epsilon)$ quantity, contradicting the weak vortex assumption which constrains $u$ to be at most $O(1)$.

(i) $0<\epsilon u<1$

This is the case of either a cyclone located on the shallow side of the escarpment or an anticyclone located on the deep side of the escarpment, and since $1+\epsilon u\left(k^{2}+1\right)^{1 / 2} \neq 0$, there are no singularities. Thus the inversion contour $C$ may be deformed back to the 


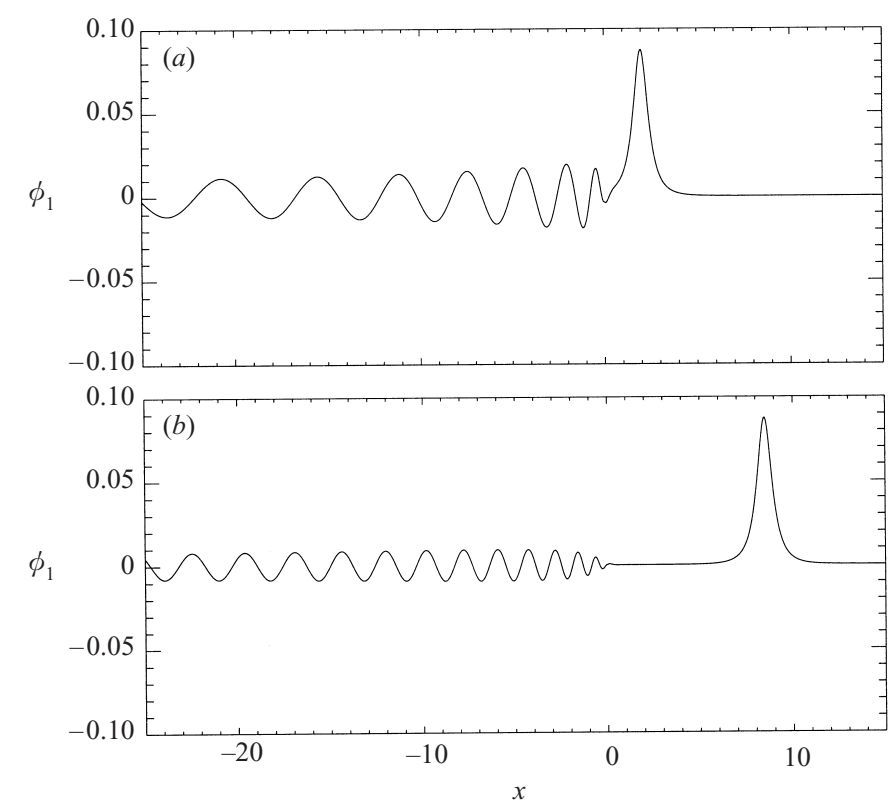

FIGURE 5. FFT evaluation of the regular solution over $y=0$, for a non-radiating weak vortex. The parameter values used are $\Gamma=-1, \epsilon=0.1$ and $L=0.5$. The times are $(a) t=7$ and $(b) t=30$.

real $k$-axis. For large $t$ the wave term is dominated by the single point of stationary phase, occurring for $-t<x<0$,

$$
k_{s}=\left((-\epsilon \xi)^{-2 / 3}-1\right)^{1 / 2},
$$

where $\xi=x / t$. The wave amplitude decays like $t^{-1 / 2}$, leaving only the steadily propagating vortex and pseudoimage as $t \rightarrow \infty$. The vortex is moving at a velocity outside the range of topographic-wave phase velocities, and in particular, since $u>0$, is moving in the opposite direction to the waves. The vortex propagates away from the wave bundle, which subsequently decays, leaving only the quasi-steady term. The transient nature of the disturbance is illustrated by fast Fourier transforms (FFT) of the regular field $\phi_{1}$ over the escarpment (i.e. at $y=0$ ) in figure 5. The large, localized disturbance near the vortex centre is the non-dispersive part of the topographic wavetrain (i.e. the pseudoimage), while the topographic waves propagate away to the west, with amplitudes decaying with time. Note that in this case there is no drift of the vortex perpendicular to the escarpment at large times.

(ii) $-1<\epsilon u<0$

This is the case of westward travelling anticyclones located on the shallow side of the escarpment or cyclones located on the deep side of the escarpment. There are simple poles in the integrals (3.37) and (3.38) at $k= \pm \gamma$, given by (3.43). At large times the solution is dominated by the behaviour near the poles, for which

$$
k \approx \frac{1}{\epsilon u} \gg 1,
$$

corresponding to short waves. Also, a simple rearrangement of (3.43) and (3.45) 


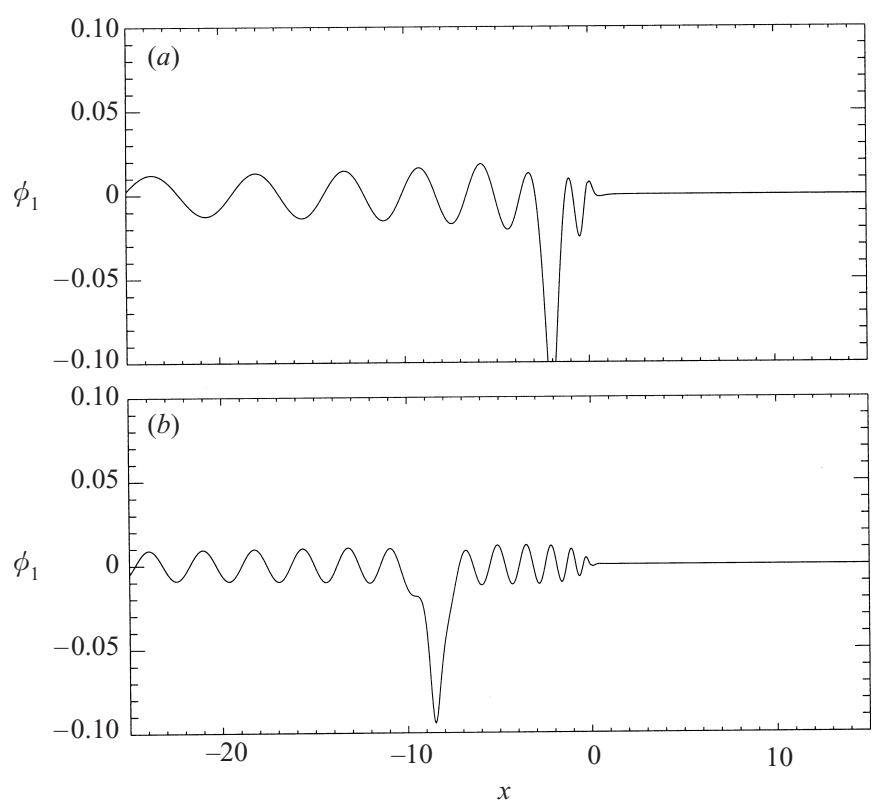

FIGURE 6. FFT evaluation of the regular solution over $y=0$, for a radiating weak vortex. The parameter values used are $\Gamma=+1, \epsilon=0.1$ and $L=0.5$. The times are $(a) t=7$ and $(b) t=30$. The non-dispersive pseudoimage is evident, and the waves ahead of it are the dispersive wave. The waves in the wake of the vortex are the steadily propagating radiated waves.

reveals

$$
u=-\frac{1}{\epsilon \sqrt{\gamma^{2}+1}}=c_{p}( \pm \gamma)
$$

since $u<0$. The asymptotic behaviour is dominated by the short waves with phase velocities close to the velocity of the vortex. This is not surprising since the vortex velocity is small, i.e. is $O(\epsilon)$, and hence the motion excites topographic waves with $c_{p}=O(\epsilon)$ which corresponds to short waves.

A residue calculation (see McDonald 1996 or Dunn 1999 for a full discussion of this calculation, and particularly of the required inversion contour) then gives the large-time response for the radiating case,

$$
\phi_{1} \approx g(y) \sin \gamma(x-u t)\left[H(x-u t)-H\left(x-c_{g} t\right)\right],
$$

where

$$
g(y)=\frac{\Gamma}{\epsilon u \gamma} \exp \left(-\frac{|y|+|L|}{|\epsilon u|}\right) .
$$

This is a wave tube existing for $u t<x<c_{g}(\gamma) t$, and whose extent along the escarpment, say $D$, grows like the difference of the phase and group velocities of the radiated waves:

$$
|D| \approx \epsilon^{-1} t\left((\epsilon u)-(\epsilon u)^{3}\right),
$$

since $\epsilon c_{g}=\epsilon c_{p}^{3}$ at $k= \pm \gamma$. Since $\epsilon^{3}$ is negligible, the rate of growth of the wave tube is approximately $u$, the phase velocity of the radiated waves. Figure 6 illustrates the process of the wave tube formation. Here, the exact linear solution $\phi_{1}$ has been evaluated from equation (3.36), over the escarpment $(y=0)$ by fast Fourier transforms. The waves ahead of the vortex decay. Note also the pseudoimage, i.e. the 
non-dispersive part of the wavetrain which propagates with the vortex. The waves are radiated from the pseudoimage, which must decrease in size, and which in turn must affect the vortex drift velocity.

In summary, in the radiating cases (anticyclone located on the shallow side or cyclone located on the deep side of the escarpment) the topographic waves decay like $t^{-1 / 2}$ except for the particular short waves with wavenumber $k= \pm \gamma$, which have phase velocity equal to the vortex zonal drift velocity. A wavetrain of finite length forms in the wake of the vortex. The trailing edge of this wave train moves at the group velocity of the radiated waves. Thus there is no disturbance for $x>c_{g} t$, and in particular the fluid is at rest at the initial position of the vortex.

Since the radiating waves have non-zero energy flux, they must exert a drag on the vortex, which in turn must respond to this loss of energy. Bell (1989) argued that a weak singular barotropic vortex moves perpendicular to a potential vorticity interface due to wave radiation. In the following section global momentum arguments are used to calculate the effect of wave radiation upon the vortex drift velocity.

\subsection{Large-time vortex trajectory}

For the case of an intense singular vortex, McDonald (1998) calculated the response of the vortex to the radiating waves by equating the energy flux of the wave tube to the rate of change of the vortex energy to derive an ODE for $L$, the distance of the vortex centre from the escarpment. In the present case an analogous equation is obtained by equating the rate of change of momentum of the vortex to the pseudomomentum (see McIntyre 1981, Dritschel 1988b; Lam \& Dritschel 2001) of the wave tube. This has the advantage that the calculations are simpler and avoids the infinite energy associated with a singular vortex; however the momentum and energy flux arguments are equivalent and yield the same result. The energy density (see e.g. Whitham 1974) in the wave tube (3.47) is

$$
\begin{aligned}
\rho & =\frac{1}{2}\left(\bar{\phi}_{1 x}^{2}+\bar{\phi}_{1 y}^{2}+\bar{\phi}_{1}^{2}\right) \\
& =\frac{\Gamma^{2}}{\gamma^{2}(\epsilon u)^{4}} \exp \left(-2 \frac{|y|+|L|}{|\epsilon u|}\right),
\end{aligned}
$$

where the overbar denotes the average over one wave period. Note that by $\phi_{1}$ in this equation is meant the large-time asymptotic solution given in (3.47). The wave power (i.e. the total energy flux), $P$, of the radiated waves is found by integrating $\rho$ over all $y$ and multiplying the result by the group velocity (Whitham 1974). The 'appropriate' group velocity is the rate of growth of the wave tube given by (3.49). This gives the wave power,

$$
P=\Gamma^{2} \mathrm{e}^{-|2 L / \epsilon u|} .
$$

The rate of change of the wave pseudomomentum parallel to the escarpment is then the wave power divided by the phase velocity,

$$
\frac{P}{\epsilon u}=\frac{\Gamma^{2}}{\epsilon u} \mathrm{e}^{-|2 L / \epsilon u|} .
$$

This quantity is also the drag on the vortex, which must respond by losing momentum. See e.g McIntyre (1981). The leading-order $x$-momentum of the vortex is, by a generalization of the result for barotropic vortices in Batchelor (1967),

$$
M_{x}=-\iint y\left(\nabla^{2} \psi-\psi\right) \mathrm{d} A=\Gamma L,
$$


the integral being taken over all space. Note that $\mathrm{d} \Gamma / \mathrm{d} t=0$ (i.e. conservation of circulation), so any change in the vortex momentum is manifested only by a change in $L$. Taking the time derivative of (3.53) and equating it with the wave momentum flux (3.52) yields the differential equation for $L$

$$
\frac{\mathrm{d} L}{\mathrm{~d} t}=\frac{\Gamma}{2 \epsilon u} \mathrm{e}^{-|2 L / \epsilon u|} .
$$

Alternatively the result (3.54) can also be obtained directly, by calculating the velocity in the $y$-direction generated by $\phi_{1}$, by differentiating (3.47) with respect to $x$ and evaluating the result at the vortex centre. Also $u<0$, so both shallow-water anticyclones and deep-water cyclones drift towards the escarpment in response to topographic wave radiation. The drift is very slow since

$$
\frac{\mathrm{d} L}{\mathrm{~d} t}=O\left(\mathrm{e}^{-1 / \epsilon}\right) .
$$

This might be expected for two reasons. First, the energy density of the radiating waves is localized near the escarpment. This is evident in the expression (3.50). Second, the wave energy is concentrated in the long topographic waves which propagate rapidly away from the vortex centre. Thus, the westward travelling vortices radiate only the less energetic, short topographic waves. This in turn is a consequence of the weak vortex assumption, which constrains the vortex velocity to be $O(\epsilon)$ on the topographicwave time scale. The topographic waves whose phase speed matches the vortex drift speed are the short waves.

It has been shown in this subsection that the pseudoimage description of the vortex motion is valid on the long advective time scale, for as long as linear theory is valid on that time scale. In the following subsection the validity of the linear theory is tested numerically by contour dynamics experiments.

\subsection{Contour dynamics results}

The choice of an infinitely long escarpment for the topography means that the method of contour dynamics is ideally suited to studying this problem. The contour initially lies along $y=0$, the interface between the shallow and deep water regions, and after the vortex is switched on at $t=0$, it evolves according to potential-vorticity conservation. The velocity field associated with the deflected contour is used to advect the vortex. The algorithm used is the quasi-geostrophic generalization of the algorithm of Dritschel (1988a). Time stepping is done by a fourth-order RungeKutta scheme $(\Delta t=0.1)$. The contour nodes are redistributed at each time step according to local curvature, and surgery, as proposed by Dritschel (1988a), has been employed at a spatial resolution of $\mu=0.15$. Several runs were repeated with a finer resolution of $\mu=0.1$ producing identical vortex trajectories and topographic contours. Also, several runs were repeated with no surgery, again resulting in identical vortex trajectories, with the exception that the number of contour nodes grew rapidly to become unmanageable.

The contour dynamics algorithm integrates the full nonlinear potential-vorticity equation (2.1) and is valid for arbitrary deformations of the initial contour. It is necessary to represent the infinite topographic contour by a finite length in the computations; importantly the contour length must be chosen so that its ends remain undisturbed during the computations. Given that the time scale employed for the contour dynamics algorithm is the advective time scale, this is hampered by the fact that the waves travel rapidly away from the vortex centre and introduce end 

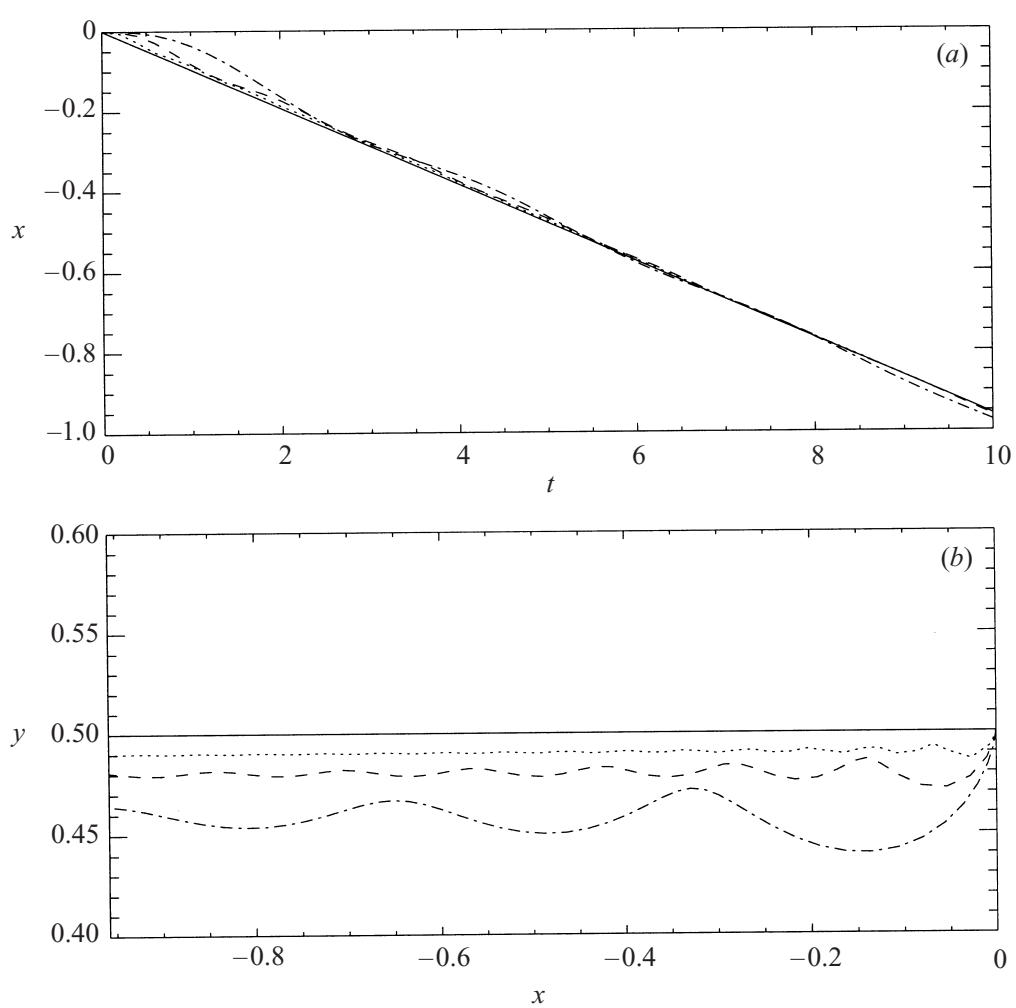

FIGURE 7. The drift of anticyclonic singular vortices, for $0<t<10$. The parameter values used are $L=0.5, \epsilon=0.1$ (dotted line), $\epsilon=0.2$ (dashed line) and $\epsilon=0.4$ (dot-dashed line). The $x$-displacement of the vortex centre is shown in $(a)$ and the path of the vortex centre in $(b)$. The solid line shows the analytic prediction, calculated from (3.26).

effects during the numerical runs. For small values of $\epsilon$ runs up to $t=10$ are possible before end effects become important, while for moderate to intense vortices much longer times are possible. Further checks on the accuracy of the algorithm include use of a vortex of zero strength, an escarpment of zero height, and an initially disturbed topographic contour in the absence of a vortex. All checks produced the correct result, and the authors are confident of the accuracy of the computations.

The main purpose of the contour dynamics investigation is to provide a check on the results of linear theory for $\epsilon \rightarrow 0$. In particular, for what range of values of $\epsilon$ is the linear theory applicable? Indeed, what is the appropriate time scale for the applicability of the linear theory? Of further interest is the effect of wave radiation on the path of the westward travelling vortices. Attention is restricted to the case $L>0$, i.e. to vortices (of both signs) located on the shallow side of the escarpment. There is no loss of generality here, since the governing equation (3.2) is invariant under the transformation

$$
\psi(x, y) \rightarrow-\psi(x,-y) .
$$

Analogous results for vortices located on the deep side of the escarpment may be deduced by symmetry. The behaviour of anticyclones differs from that of cyclones, and each case is treated separately. 

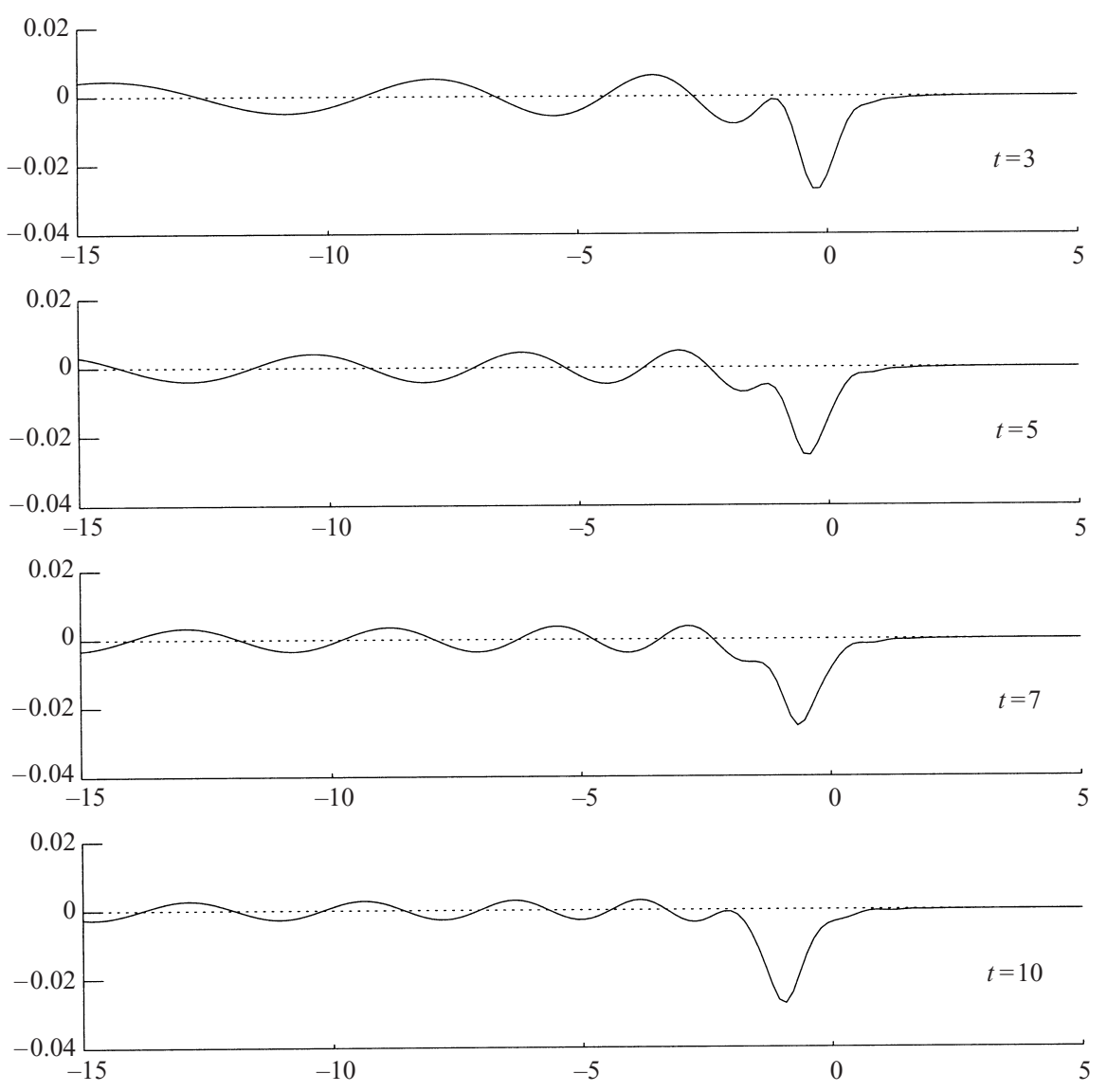

FIGURE 8. The evolution of the contour for a weak anticyclone. The parameter values used are $L=0.5, S=10$ (i.e. $\epsilon=0.1$ ). Note the small-amplitude disturbance, and the non-dispersive pseudoimage.

\subsubsection{Anticyclones}

Experiments were carried out for $\epsilon=0.1,0.2$ and 0.4 , with $L=0.5$ in each case. Figure $7(a)$ shows a plot of the vortex zonal drift velocity compared with the linear theory prediction given in equation (3.26). It is apparent that the prediction of the zonal drift agrees well with the numerical results, even up to $\epsilon=0.4$. Figure 7(b) shows a plot of the vortex trajectory, again compared with the linear theory prediction. Note the initial southward movement in all cases presented. This observation is consistent with the linear theory, which predicts that anticyclones initially move south as the result of the establishment of a secondary dipole (see the earlier discussion). The maximum meridional drift of the vortex differs by about $10 \%$ from that predicted by linear theory for $\epsilon=0.4$. It is apparent that the linear theory makes a more accurate prediction of the meridional drift as the parameter is decreased. This is to be expected as the linear theory is formally only valid in the limit of vanishing $\epsilon$, and it must be concluded that there are slight nonlinear effects for the values used in the contour dynamics simulations.

Figure 8 shows a plot of the evolution of the contour for $\epsilon=0.1$. The westward travelling dispersive waves are evident. Note also the disturbance which propagates with the vortex, which consists of fluid originally located on the shallow side of the 

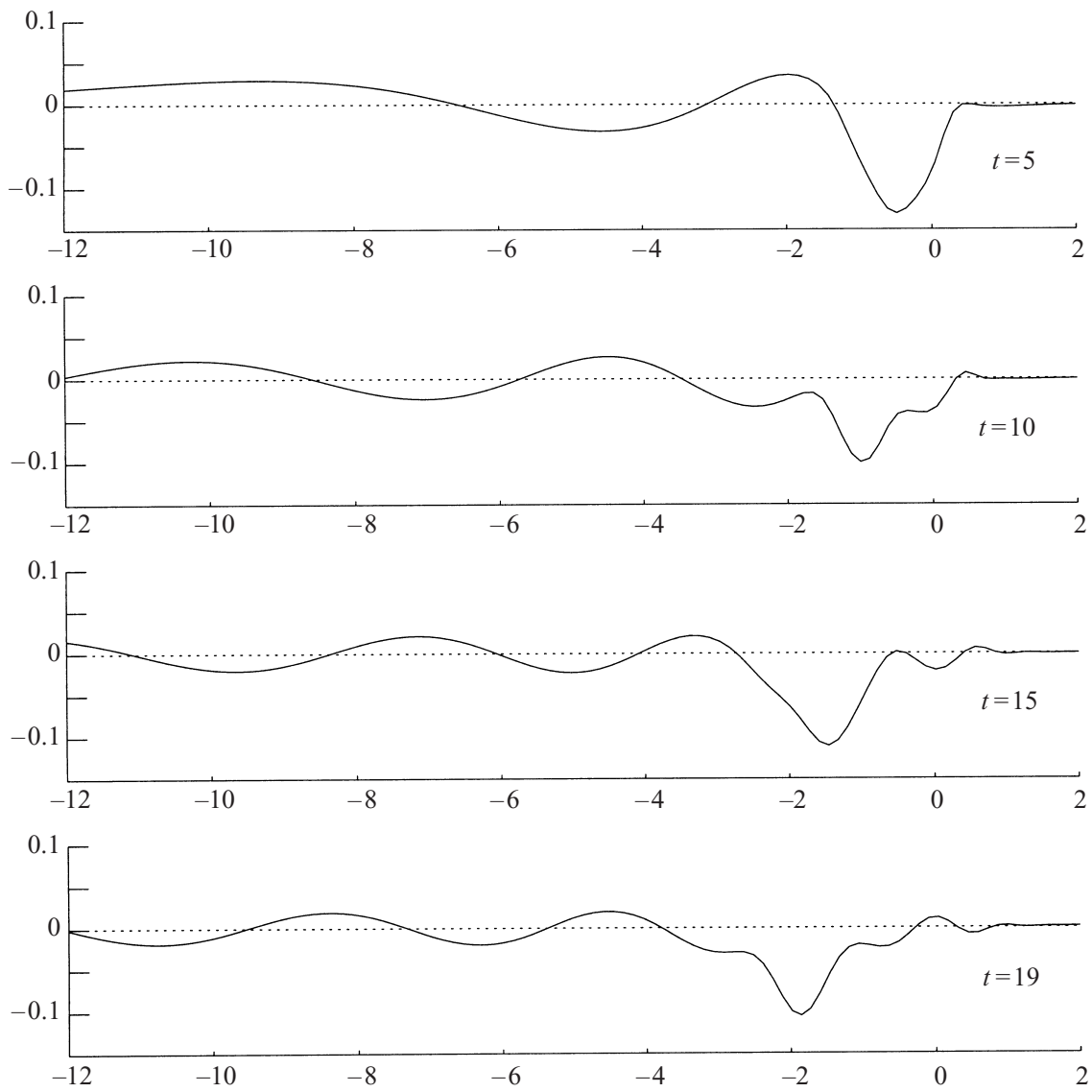

FIGURE 9. As figure 8, except $\epsilon=0.4$.

escarpment, and has crossed the escarpment to the deep side gaining net cyclonic relative vorticity of magnitude $2 \epsilon^{-1}$. In the limit of small $\epsilon$ the circulation of this patch has precisely the correct magnitude to advect the primary vortex in the sense of its pseudoimage in the escarpment. Put another way, the steadily propagating, non-dispersive 'pulse' of cyclonic relative vorticity is the pseudoimage. There is no visible evidence of topographic wave radiation in this plot, and this observation is reinforced by the vortex drift, which after the initial southward movement is predominantly zonal. This may be expected as the wave radiation and subsequent zonal drift predicted by the theory is too weak to be evident in the plots. Moreover end effects in the contour prevent runs long enough to observe any significant wave radiation.

However, consider figure 9, which shows the evolution of the contour for $\epsilon=0.4$. Here, the topographic waves and the pseudoimage are also apparent. Note the radiating waves, of larger amplitude than for the weaker vortex, in the wake of the pseudoimage. This behaviour is qualitatively the same as that shown in the FFT plot of the analytical solution shown in figure 6. Enhanced meridional drift is the response of the vortex to topographic wave radiation - see figure 7. Given that the vortex is singular, and so cannot change its shape, escarpment-ward meridional drift is the only response that the vortex can have to wave radiation. 

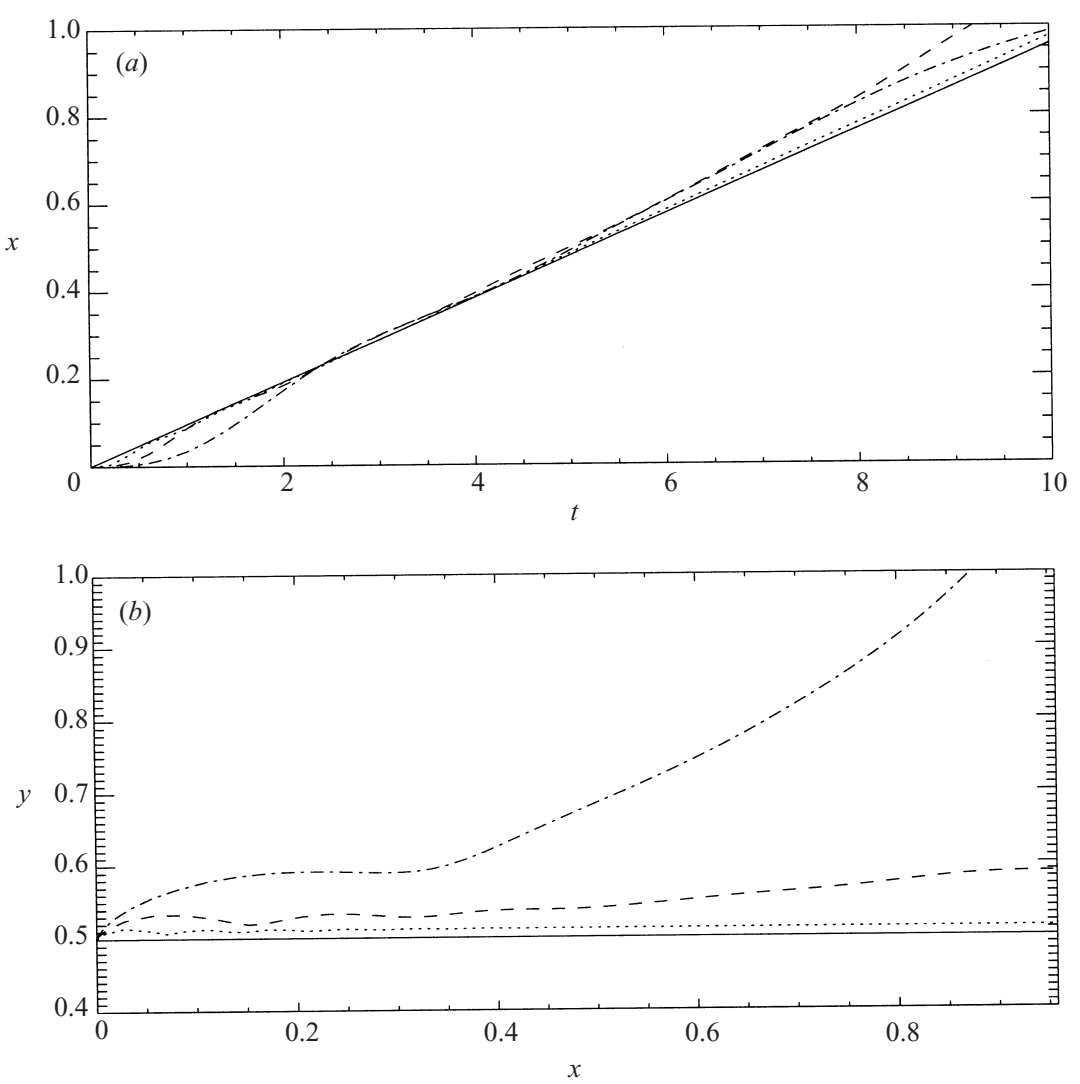

Figure 10. The drift of cyclonic singular vortices. The parameter values used are $L=1, \epsilon=0.1$ (dotted line), $\epsilon=0.2$ (dashed line) and $\epsilon=0.4$ (dot-dashed line). The $x$-displacement of the vortex centre is shown in $(a)$ and the path of the vortex centre in $(b)$. The solid line shows the analytic prediction, calculated from (3.26).

\subsubsection{Cyclones}

Again experiments were carried out for $\epsilon=0.1,0.2$ and 0.4 , with $L=0.5$ in each case. For cyclones the theory predicts that there will be no steady wave radiation in the wake of the vortex. Figure 10(a) shows a plot of the vortex zonal drift velocity compared with the linear theory prediction given in equation (3.26). The prediction is in good agreement with the numerical results for small values of $\epsilon$, but it is evident that the linear theory predicts the vortex motion for a smaller range of values of $\epsilon$ for cyclones than it does for anticyclones. This statement is reinforced in figure $10(b)$, which shows the trajectory of the vortex centre. For $\epsilon=0.4$ the cyclone has undergone substantial meridional drift. This cannot be the result of wave radiation, since the cyclone propagates in the opposite direction to the topographic waves. Therefore, the meridional drift must be the result of nonlinear effects.

To understand why nonlinear effects are more pronounced for cyclones than anticyclones consider figure 11, a plot of the evolution of the contour for $\epsilon=0.1$. As for the anticyclones the dispersive topographic waves are evident. The pseudoimage is also evident, this time consisting of a patch of fluid which had crossed the escarpment from deep to shallow water, gaining net anticyclonic vorticity, and which, in the limit $\epsilon \rightarrow \infty$, has precisely the right circulation to advect the cyclone as if the escarpment were a plane wall. 

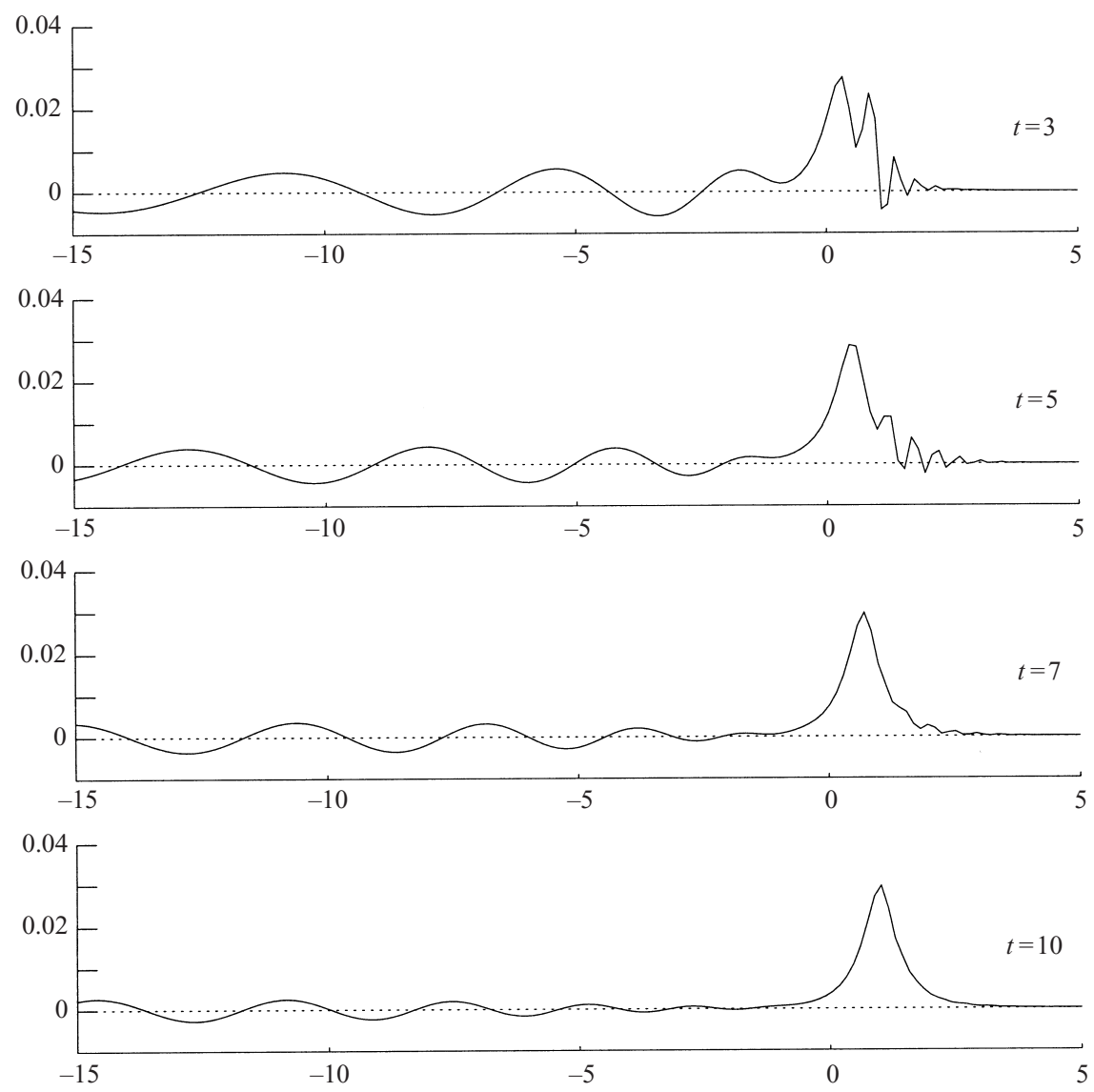

FIGURE 11. The evolution of the contour for a weak cyclone. The parameter values used are $L=0.5, S=10$ (i.e. $\epsilon=0.1$ ).

Next consider figure 12, which shows the contour evolution for a cyclone with $\epsilon=0.4$, and illustrates a fundamental difference between a weak cyclone and a weak anticyclone. The initial disturbance 'wants' to move west, dispersing as topographic waves. However, in this case the sense of the vortex circulation is such as to counter this tendency and is sufficiently strong to prevent the initial disturbance from moving away from the vicinity of the vortex. In contrast an anticyclone reinforces the westward propagation of the initial disturbance. Figure 12 shows a dipole consisting of the primary, cyclonic vortex together with the newly formed anticyclonic relative vorticity, formed by fluid crossing the escarpment. By $t \approx 6$ this dipole propagates northeast, away from the escarpment. It will be shown below that for moderate intensity cyclones located on the shallow side of the escarpment, the formation of dipoles is a generic feature.

\subsection{Discussion}

It has been shown that, in the limit $\epsilon \rightarrow 0$, linear theory predicts that a weak singular vortex near an escarpment drifts in the sense of its image in the escarpment. While an explicit definition of a pseudoimage is new, the phenomenon has been noted in two previous works. Bell (1989) and Stern \& Flierl (1987) investigated the motion of a singular barotropic vortex near a potential vorticity interface and a shear flow 

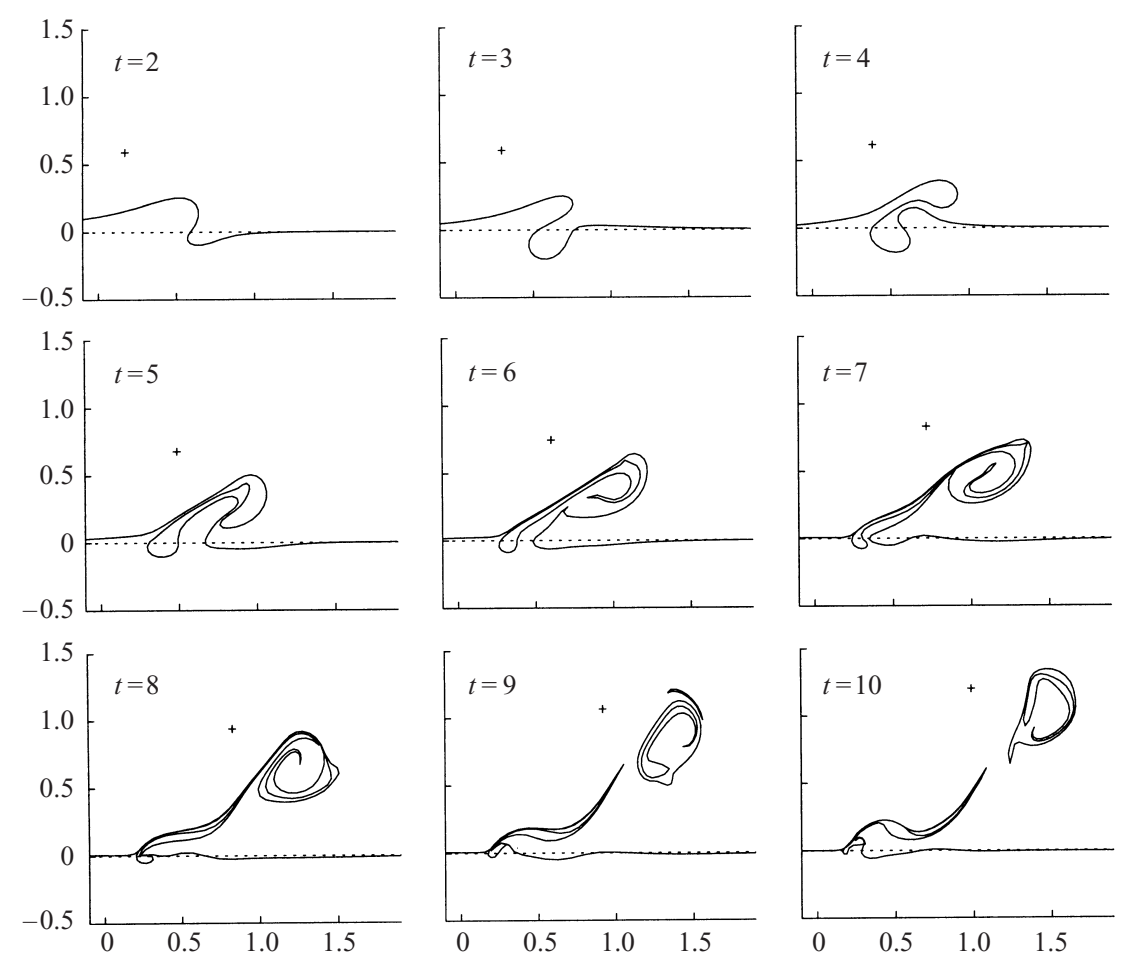

FIGURE 12. As figure 11, except $\epsilon=0.4$. The vortex centre is indicated by the cross.

respectively, and found that weak vortices move parallel to the interface in the image sense.

Westward travelling anticyclones (resp. cyclones) located on the shallow (deep) side of the escarpment radiate short topographic waves as $t \rightarrow \infty$. Global momentum arguments were used to estimate the meridional drift of the vortex centre due to the wave radiation. This drift is exponentially slow since the energy flux associated with the short waves is small. It should be emphasized that meridional drift is the only possible response of a singular vortex to wave radiation, since its shape is fixed.

Contour dynamics experiments have shown that for small $\epsilon$, linear theory accurately describes the drift of the vortex centre even beyond its strict range of validity, which is formally $t \ll O\left(\epsilon^{-1}\right)$. Both anticyclones and cyclone drift parallel to the escarpment at the velocity due to the pseudoimage. The physical meaning of the pseudoimage was identified as a non-dispersive patch of relative vorticity in the deflected topographic contour.

For anticyclones located on the shallow side of the escarpment, linear theory is accurate up to $\epsilon=0.4$ and beyond. The wave-induced meridional drift of the vortex centre increases with increasing $\epsilon$, and the amplitude of the radiated waves also increases with $\epsilon$.

On the other hand it has been shown that nonlinear effects are important for cyclones at smaller values of $\epsilon$. The primary mechanism for the breakdown of the linear theory for cyclones is the accumulation of anticyclonic relative vorticity near the vortex centre. This is a result of the circulation of the primary vortex driving fluid against the preferred direction of the topographic waves. This anticyclonic relative vorticity is then able, through a dipole mechanism, to advect the primary vortex 


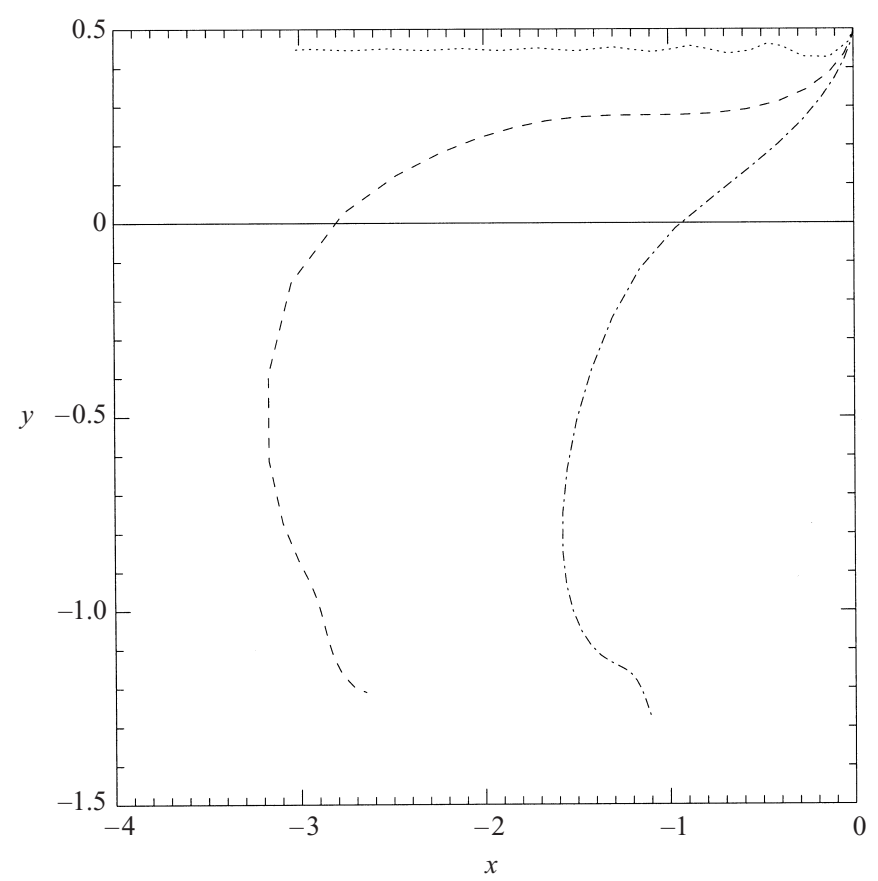

FIGURE 13. A plot of the trajectories for the moderate intensity anticyclones for $0<t<40$. The parameter values used are $L=0.5, S=2$ (dotted line), $S=1$ (dashed line) and $S=0.5$ (dot-dashed line).

northeast. At large times the cyclone leaves the vicinity of the escarpment, a process which Zavala Sanson et al. (2000) refer to as 'back-reflection'. A more thorough discussion of this phenomenon is given below, in the investigation of the behaviour of moderate intensity vortices.

\section{A moderate singular vortex: contour dynamics results}

To complete the study of the motion of a singular vortex near an escarpment, contour dynamics results are presented in this section for moderate vortices. There is no theory available for the $S \approx 1$ regime, but interesting results are anticipated. This is particularly so when it is realized that intense vortices propagate 'west', regardless of the sign of the vortex, but weak vortices propagate in opposite directions according to their pseudoimage. In which direction, therefore, do moderate vortices drift? Attention is restricted to the cases $S=2$ (moderately weak vortex), $S=1$ (moderate vortex) and $S=0.5$ (moderately intense vortex). Once again only vortices located on the shallow side of the escarpment are considered and the cases of anticyclones and cyclones are considered separately.

\subsection{Anticyclones}

Figure 13 shows a plot of the trajectories of the moderate anticyclones. For $S=2$, the moderately weak anticyclone appears to still be modelled well by the linear theory. At large times the drift is purely zonal. For the moderate anticyclone $(S=1)$, the initial response also appears to be linear. However the vortex centre later turns south and crosses the escarpment, traversing a large arc before finally turning east. The moderately intense anticyclone $(S=0.5)$ also moves sharply south, crosses the 


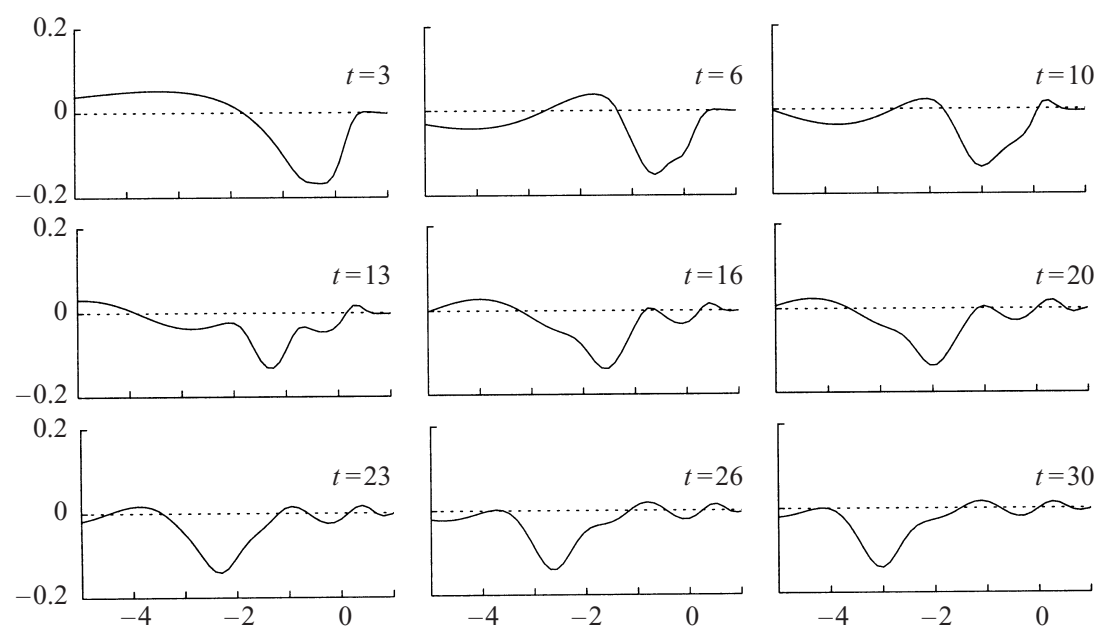

FIGURE 14. Evolution of the contour for an anticyclone with $L=0.5$ and $S=2$.
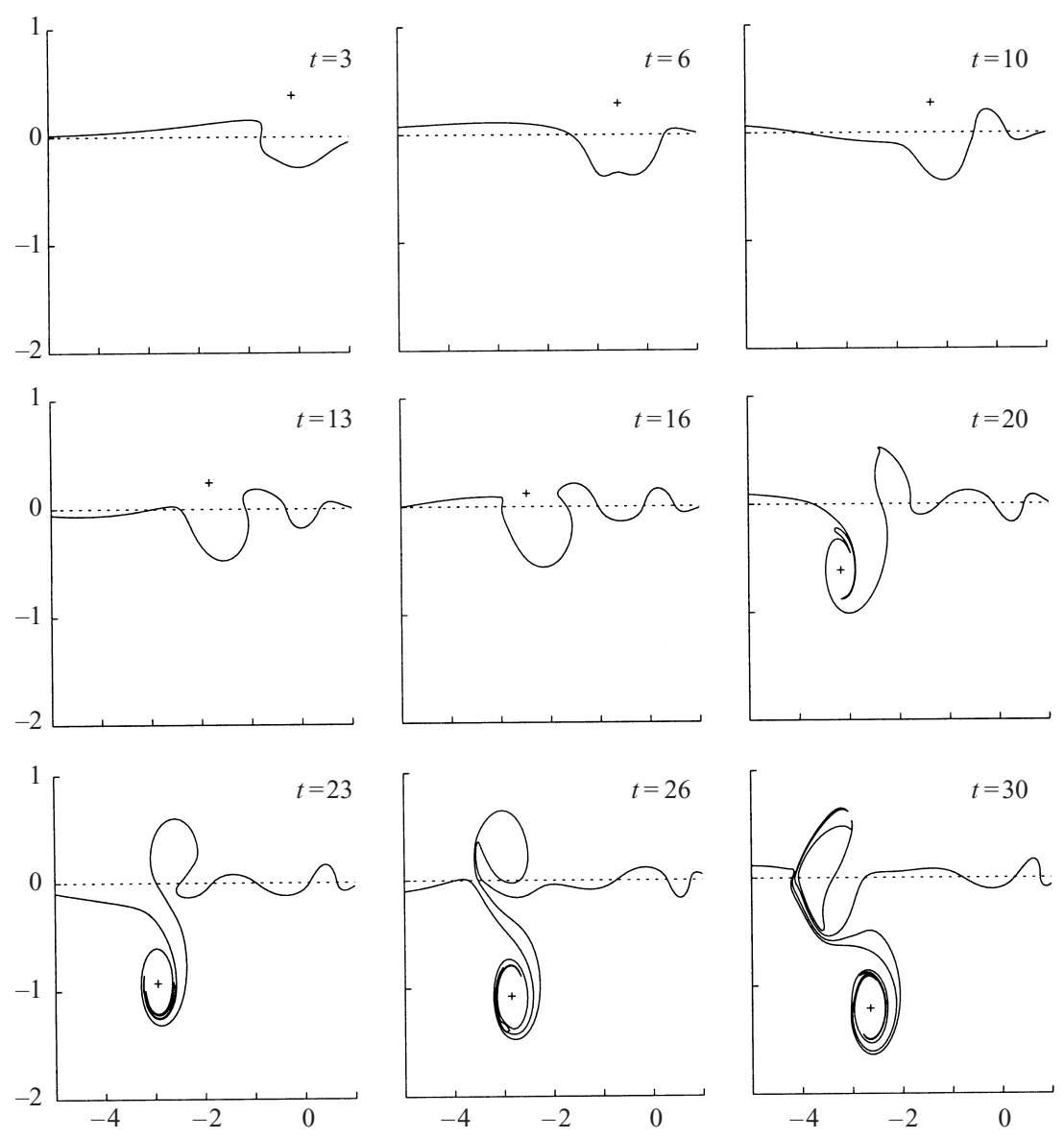

Figure 15. As figure 14 except $S=1$. 

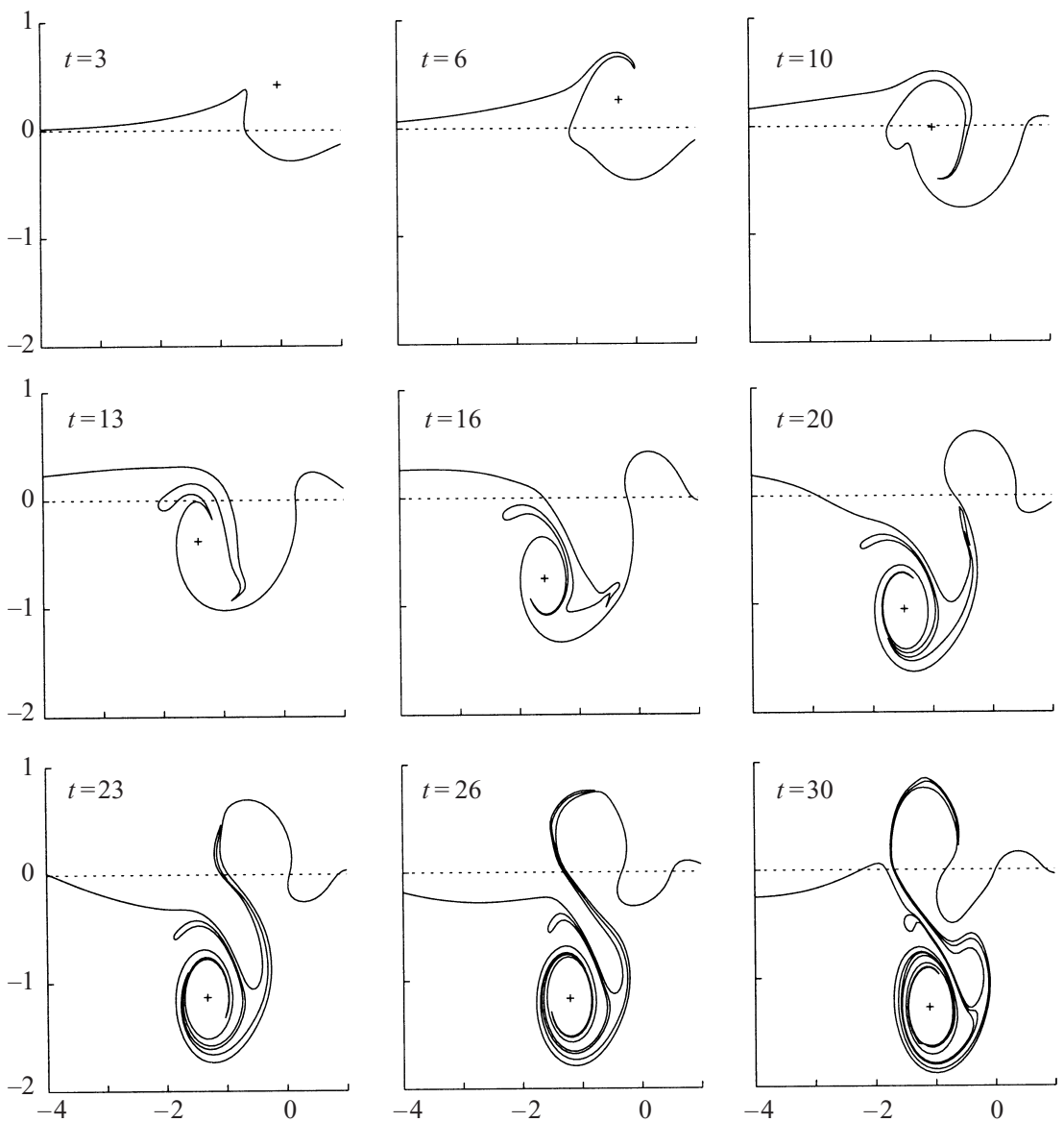

FIgURE 16. As figure 14 except $S=0.5$.

escarpment and turns east at later times, with the exception that the initial westward displacement is much reduced.

Figure 14 shows the contour evolution for $S=2$. The response does indeed appear linear. The pseudoimage and radiated waves are apparent. It must be concluded that linear theory predicts the motion of anticyclones even for $S=2$ (i.e. $\epsilon=0.5$ ), for at least forty eddy turnover times.

Next consider figure 15. At short times the topographic waves are linear and the vortex centre is advected by the pseudoimage. The wave radiation appears strong enough to cause the vortex to reach and cross the escarpment. At this stage the vortex begins to wrap the topographic contour, and has a small but significant patch of cyclonic relative vorticity nearby. The dipole mechanism then turns the vortex from its westward drift and the vortex centre moves east at large times.

Figure 16 shows the evolution of the contour for $S=0.5$. At short times the vortex wraps the contour up, and then crosses the escarpment. As for the previous case a patch of cyclonic relative vorticity accompanies the vortex in its evolution and the dipole mechanism turns the vortex east at later times, but more sharply than for the $S=1$ case, since the relative vorticity acquired by fluid crossing the escarpment is greater. 


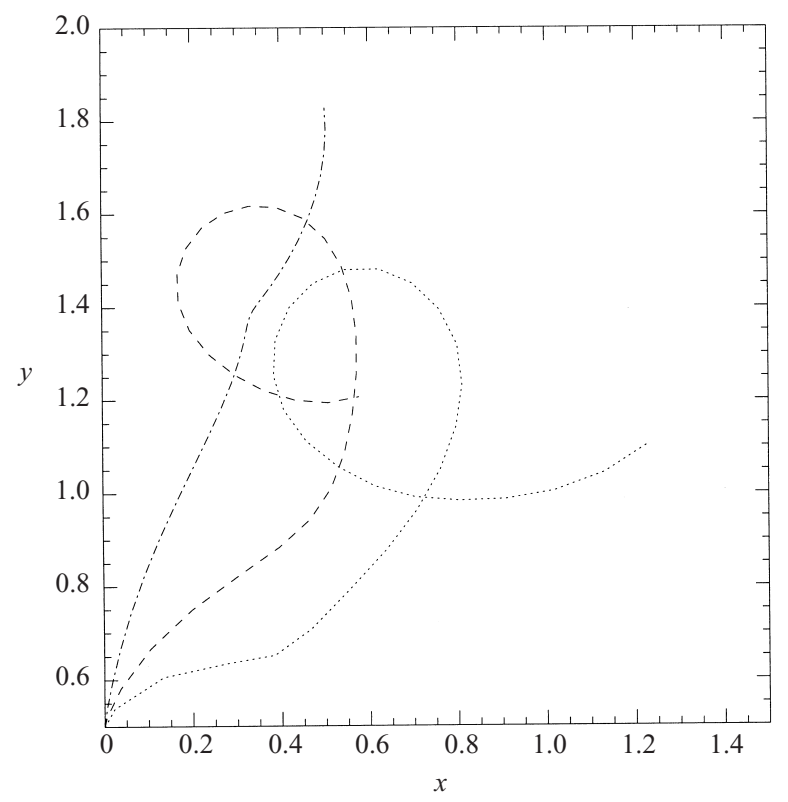

FIGURE 17. A plot of the trajectories for the moderate intensity cyclones for $0<t<30$. The parameter values used are $L=0.5, S=2$ (dotted line), $S=1$ (dashed line) and $S=0.5$ (dot-dashed line).

\subsection{Cyclones}

Figure 17 shows a plot of the trajectories of the moderate cyclones. For $S=2$, the moderately weak cyclone follows a generally northeast path. For the moderate anticyclone $(S=1)$, the initial motion is also northeast, but path of the vortex centre then 'loops'. However the net migration of the vortex centre is north and east. The moderately intense $(S=0.5)$ cyclone exhibits the same behaviour except that the drift is more east and less north.

Figures 18-20 show the contour evolution for the moderate cyclones. The behaviour is qualitatively the same in all cases. The cyclone pinches off some of the topographic contour. This anticyclonic relative vorticity together with the primary cyclonic vortex form a dipole, and the general migration of the dipole is northeast. In particular the vortex propagates away from the escarpment, and is only affected by the anticyclonic patch at later times. The looping motion is characteristic of a dipole composed of circulations of differing magnitude.

\subsection{Discussion}

Contour dynamics experiments have shown that for moderate singular vortices the characteristic of the motion is the formation of dipoles, and curiously, both cyclones and anticyclones drift east at large times. The process by which this happens differs slightly for anticyclones compared with cyclones. An anticyclone crosses the escarpment. A large patch of fluid initially located on the shallow side of the escarpment accompanies the vortex as is crosses the escarpment. This fluid gains cyclonic relative vorticity, and forms a dipole with the primary vortex. The dipole mechanism curves the path of the vortex to the east at large times. On the other hand cyclones draw fluid from the deep side of the escarpment. This fluid develops anticyclonic relative vorticity, and the dipole formation proceeds more quickly than the case of anticyclones. The cyclones move northeast from the outset, and at large times move away from the 

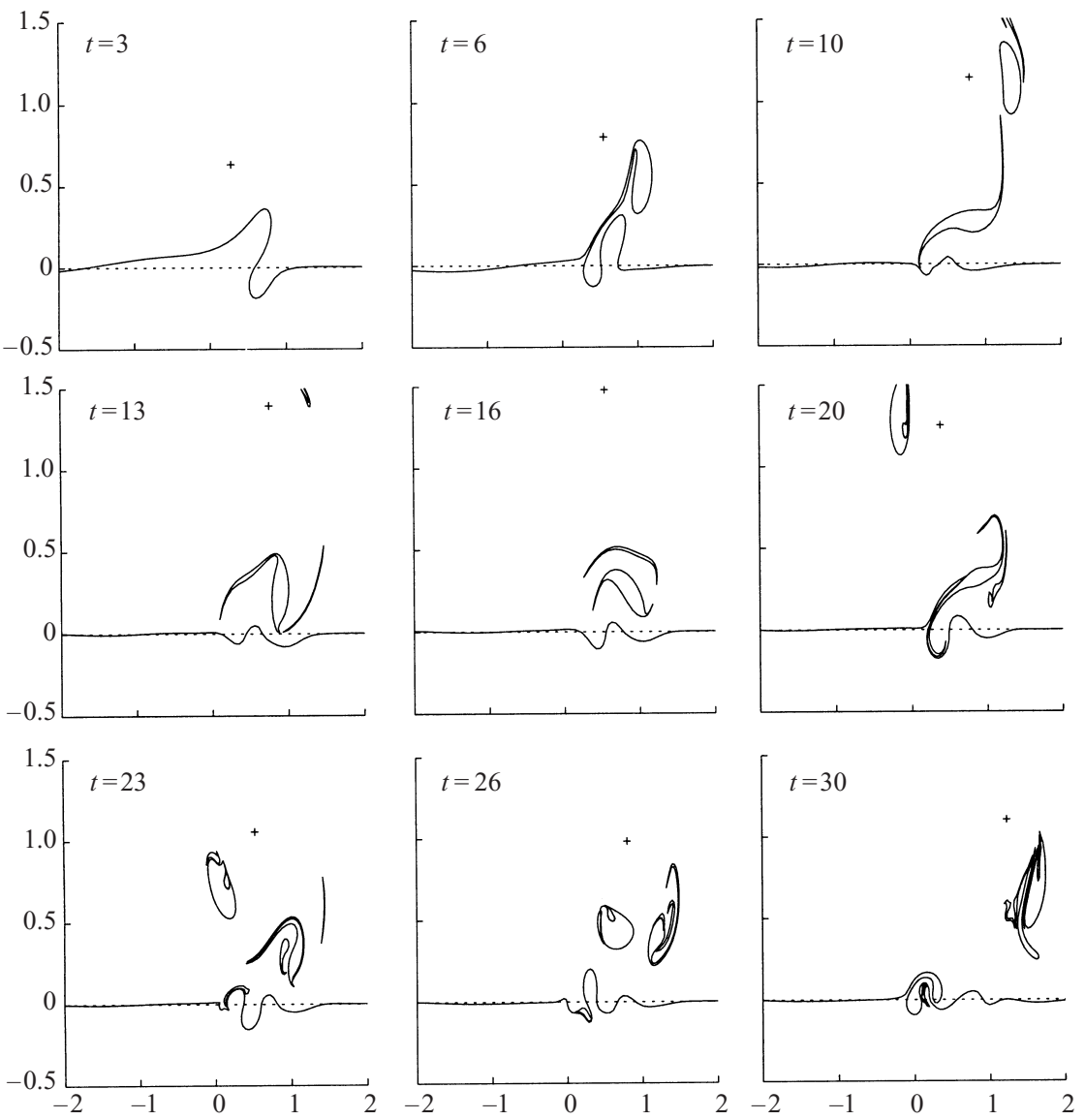

FIGURE 18. Evolution of the contour for a cyclone with $L=0.5$ and $S=2$.

escarpment. The process of dipole formation occurs for cyclones over a larger range of parameter values than for anticyclones. This was indicated in the discussion of the weak cyclones, where, even for relatively large values of $S$ the circulation of the vortex competes with the topographic wave mechanism, causing a buildup of anticyclonic relative vorticity near the vortex centre.

The results of this subsection can be used to explain aspects of the experimental results of Zavala Sanson et al. (2000) who have recently investigated the behaviour of barotropic vortices near an escarpment, both experimentally and numerically. In that investigation the escarpment lay in the meridional direction on a $\beta$-plane. The $\beta$-effect was small in comparison to the topography, and mainly served to bring a vortex near to the escarpment. The strength of the vortices was of the same order of magnitude as the relative vorticity produced by fluid crossing the escarpment, or, in the terminology of the present work, $S \approx 1$. It was found that anticyclones situated on the shallow side of the escarpment were able to 'climb' the topographic gradient, while cyclones were 'back-reflected' due to dipole formation, precisely the behaviour observed in these contour dynamics experiments. The dipole mechanism is sufficiently strong to overcome the westward drift due to $\beta$. In a similar vein, McDonald \& Dunn (2000) used contour dynamics to investigate the motion of a singular vortex near a seamount (in the present context, an escarpment may be considered to be the 

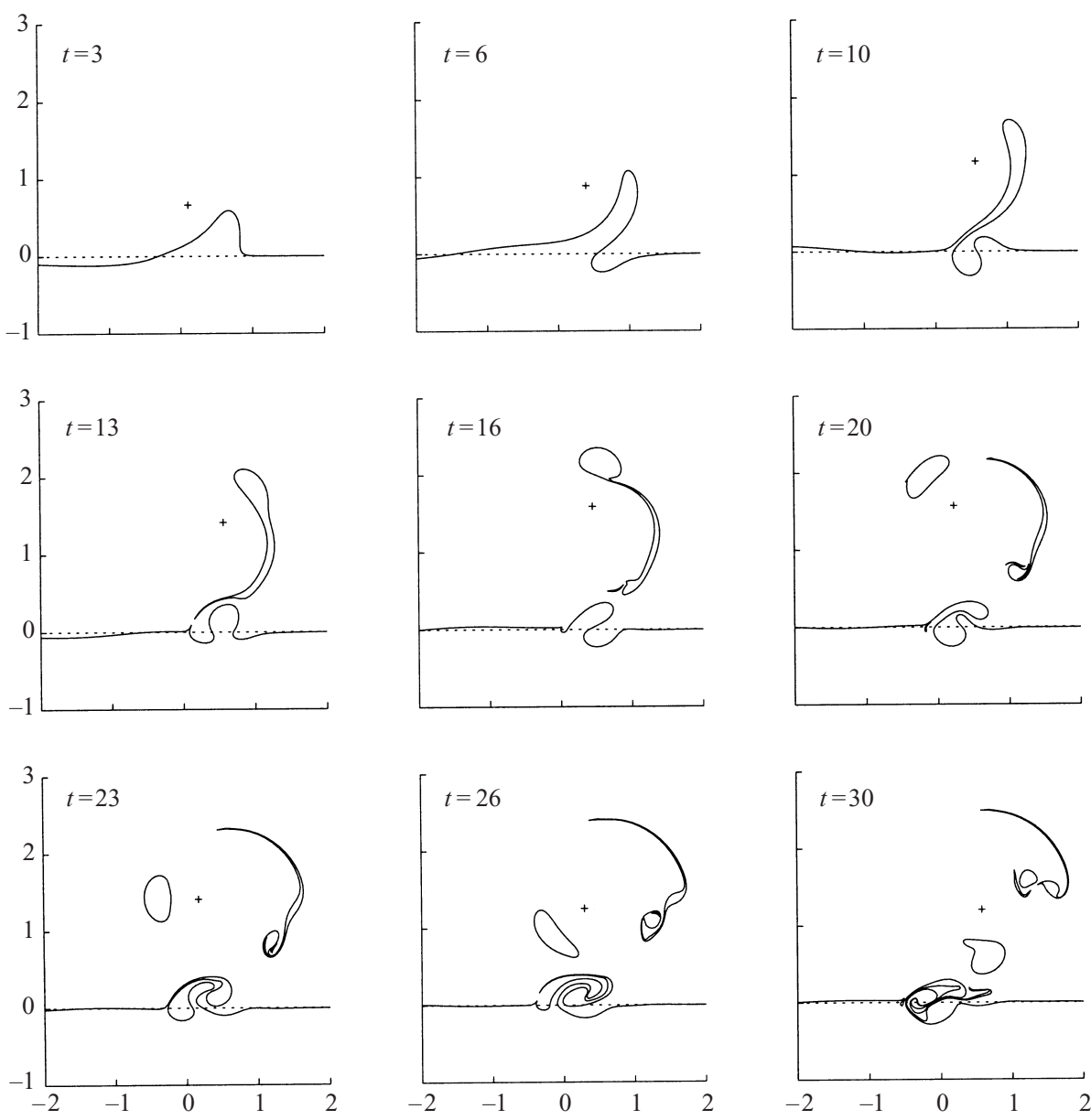

Figure 19. As figure 18 except $S=1$.

limit of a seamount with infinite radius), and found that dipole formation is again a characteristic of the motion for moderate strength vortices.

Lam \& Dritschel (2001) find that maximum meridional displacement of a circular vortex on a $\beta$-plane occurs for moderate intensity vortices. They describe the mechanism for this process in terms of a 'trailing eddy', i.e. a part of the radiated Rossby wavetrain that has circulation in the opposite sense to the primary vortex. Put another way, for the moderate intensity $\beta$-plane vortex, a dipole forms between the primary vortex and the shed vorticity. Again, this is precisely the same mechanism that has been observed in the present case. It might be expected that the robust dipole formation of the type described here is a frequently occurring phenomenon. For example, dipole formation maybe extremely important in cross-frontal mixing see for example Spall \& Chapman (1998).

\section{Conclusions}

In this paper a study of the motion of an intense singular vortex near an escarpment has been carried out for the full range of values of vortex intensity. Several important conclusions may be drawn. 

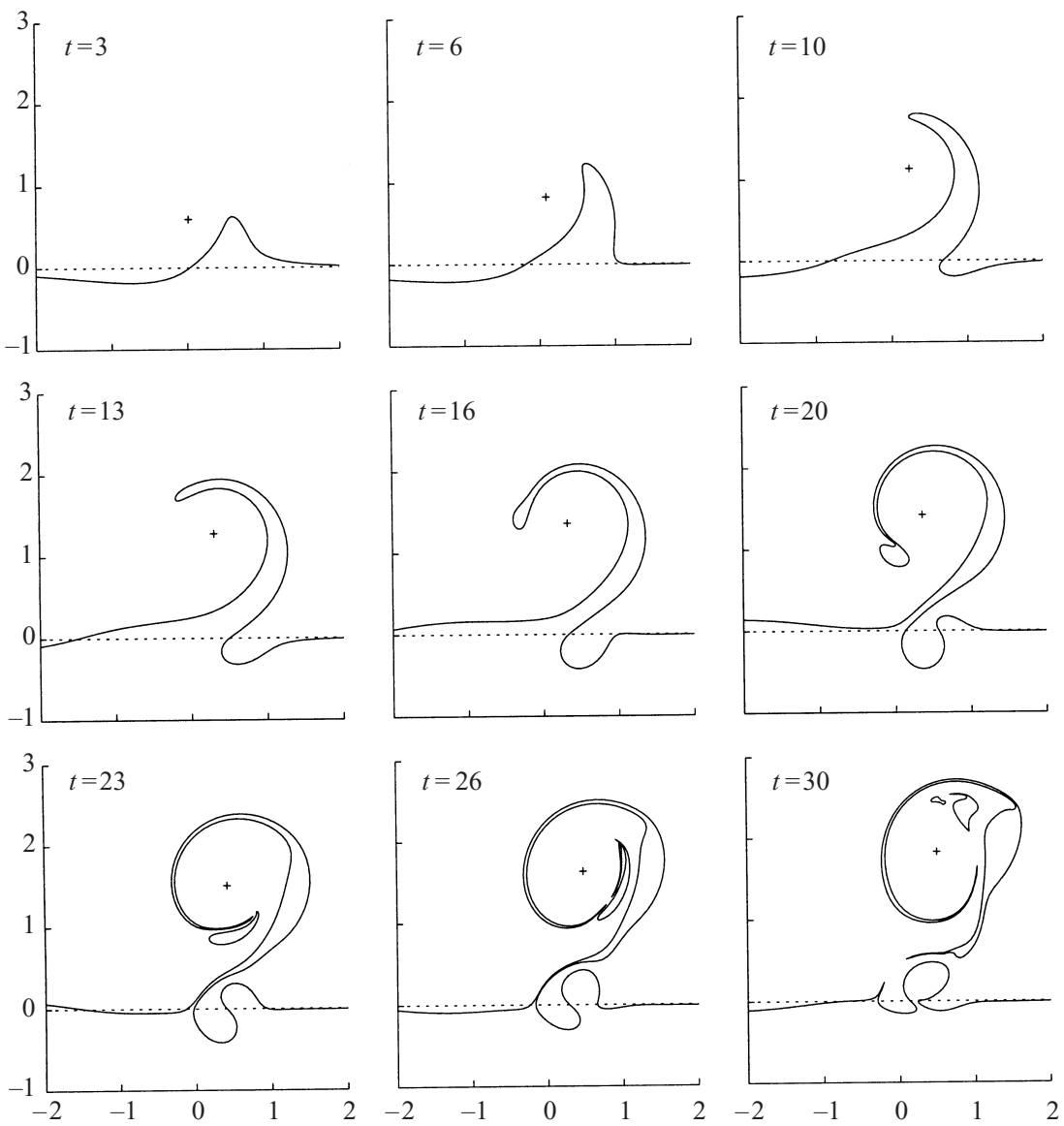

FIGURE 20. As figure 18 except $S=0.5$.

First, analytic results for a weak vortex have predicted that the escarpment acts like a plane wall. This phenomenon was dubbed the 'pseudoimage of the vortex', since there is no true image vortex. In the case where the vortex travels in the same direction as the topographic waves, expressions for vortex drift induced by wave radiation and based on pseudomomentum arguments were found. This drift is purely meridional and negligible for times of order unity. Contour dynamics confirmed the analytical predictions and revealed that the pseudoimage is a steadily propagating, non-dispersive, patch of relative vorticity in the deflected topographic contour. It might be anticipated that more realistic models of weak vortices near an escarpment, or indeed any sharp topographic gradient such as a seamount, might evolve in the sense of its image in the potential-vorticity interface.

Second, contour dynamics show that dipole formation is generic for moderate intensity vortices. This is in keeping with experimental results, and directly analogous to numerically observed dynamics of a vortex on the $\beta$-plane. Dipole formation is important since these structures are robust, stable, nonlinear and are able to travel rapidly. Hence once formed they will dominate the ensuing dynamics, and as is wellknown this characteristic plays an important role in the transport of passive scalars such as salt, heat or biota.

Third, there are several aspects of physical vortices which the model does not 
account for. For example, changes in the shape of the vortex may be of great importance to the dynamics, and as a first step in understanding the evolution of a continuously distributed vortex near an escarpment, an initially circular patch of uniform relative vorticity is considered in Dunn (1999) and work by him currently underway. Further aspects to be addressed in future studies include more complex topography, the relaxation of the quasi-geostrophic assumption, the effects of the vertical structure of the vortex and the effect of friction.

The authors dedicate this paper to the memory of trusted friend and colleague, Rupert Ford, who will be sadly missed, and whose significant contribution to this work as the $\mathrm{PhD}$ examiner of D.C.D. is gratefully acknowledged.

The authors are grateful to the referees for comments which have improved this paper.

\section{REFERENCES}

Ambramowitz, M. \& Stegun, I. A. (Eds) 1972 Handbook of Mathematical Functions. Dover.

Batchelor, G. K. 1967 Introduction to Fluid Dynamics. Cambridge University Press.

Beismann, J. D., KäsE, R. H. \& Lutejeharms, J. R. E. 1991 On the influence of submarine ridges on translation and stability of Agulhas rings. J. Geophys. Res. E 104(C4), 7897-7906.

BELL, G. I. 1989 Interaction between vortices and waves in a simple model of geophysical flow. Phys. Fluids A 2, 575-586.

Bender, C. M. \& Orszag, S. A. 1978 Advanced Mathematical Methods for Scientists and Engineers. McGraw-Hill.

DritsCheL, D. G. 1988a Contour surgery: a topological reconnection scheme for extended integrations using contour dynamics. J. Comput. Phys. 77, 240-266.

DrITSCHEL, D. G. $1988 b$ Nonlinear stability bounds for inviscid, two-dimensional, parallel or circular flows with monotonic vorticity, and the analogous three-dimensional quasi-geostrophic flows. J. Fluid Mech. 191, 575-582.

Dritschel, D. G. \& Ambaum, M. H. P. 1997 A contour-advective semi-Lagrangian numerical algorithm for simulating fine-scale conservative dynamical fields. Q. J. R. Met. Soc. 123, 1097 1130 .

DunN, D. C. 1999 Vortex interactions with topographic features in geophysical fluid dynamics. PhD thesis, University College London.

Firing, E. \& BeARdSley, R. 1976 The behaviour of a barotropic eddy on a $\beta$-plane. J. Phys. Oceanogr. 6, 57-65.

FLIERL, G. R. 1977 The application of linear quasi-geostrophic dynamics to Gulf Stream rings. J. Phys. Oceanogr. 7, 365-379.

FLIERL, G. R. 1984 Rossby wave radiation from a strongly nonlinear warm eddy. J. Phys. Oceanogr. 14, 47-58.

Gradshteyn, I. S. \& Rhyzik, I. M. 1980 Tables of Integrals Series and Products. Academic.

JoHNSON, E. R. 1984 Starting flow for an obstacle moving transversely in a rapidly rotating fluid. J. Fluid Mech. 149, 71-88.

Johnson, E. R. 1985 Topographic waves and the evolution of coastal currents. J. Fluid Mech. 160, 499-509.

Johnson, E. R. \& DaveY, M. K. 1990 Free-surface adjustment and topographic waves in coastal currents. J. Fluid Mech. 219, 273-289.

LAM, J. S.-L. \& Dritschel, D. G. 2001 On the beta-drift of an initially circular vortex patch. J. Fluid Mech. 436, 107-129.

Lighthill, M. J. 1974 Waves in Fluids. Cambridge Unversity Press.

McDonald, N. R. 1992 Topographic dispersal of bottom water. J. Phys. Oceanogr. 23, 954-969.

McDonald, N. R. 1996 Topographic wave radiation and modon decay. Geophys. Astrophys. Fluid Dyn. 83, 51-77.

McDonald, N. R. 1998 Motion of an intense vortex near topography. J. Fluid Mech. 367, 359-377. 
McDonald, N. R. \& Dunn, D. C. 1999 Some interactions of a vortex with a seamount. Il Nuovo Cimento 22C, 885-898.

MCIntyre, M. E. 1981 On the wave momentum myth. J. Fluid Mech. 106, 331-347

McWilliams, J. C. \& Flierl, G. R. 1979 On the evolution of isolated, nonlinear vortices. J. Phys. Oceanogr. 9, 1155-1182.

Meleshio, V. V., Konstantinov, M. Y., Gurzhi, A. A. \& Konovalijuk, T. P. 1992 Advection of a vortex pair atmosphere in a velocity field of point vortices. Phys. Fluids A 4, 2779-2797.

Mied, R. S. P. \& Lindeman, G. J. 1979 The propagation and evolution of cyclonic Gulf Stream rings. J. Phys. Oceanogr. 9, 1183-1206.

Reznik, G. M. 1992 Dynamics of singular vortices on a beta-plane. J. Fluid Mech. 240, 405-432.

Reznik, G. M., Grimshaw, R. \& Benilov, E. S. 2000 On the long term evolution of an intense localised divergent vortex on the $\beta$-plane. J. Fluid Mech. 422, 249-280.

Richardson, P. L. \& Tychensky, A. 1998 Meddy trajectories in the Canary basin. J. Geophys. Res. 103, 25029-25045.

Spall, M. A. \& Chapman, D. C. 1998 On the efficiency of baroclininc heat transport across narrow fronts. J. Phys. Oceanogr. 28, 2275-2287.

STERN, M. E. 2000 Scattering of an eddy advected by a current towards a topographic obstacle. $J$. Fluid Mech. 402, 211-223.

Stern, M. E. \& Flierl, G. R. 1987 On the interaction of a vortex with a shear flow. J. Geo. Res. 92, 10733-10744.

Sutyrin, G. G. \& Flierl, G. R. 1994 Intense vortex motion on the beta plane: development of the beta gyres. J. Atmos. Sci. 51, 773-790.

Whitham, G. B. 1974 Linear and Nonlinear Waves. Wiley.

Zavala Sanson, I., van Heisst, G. J. F. \& Doorschoot, J. J. J. 2000 Reflection of barotropic vortices from a step-like topography. Il Nuovo Cimento 22C, 909-930. 